Acta Crystallographica Section A

Foundations of Crystallography

ISSN 0108-7673

Received 16 April 2009

Accepted 10 November 2009

C) 2010 International Union of Crystallography Printed in Singapore - all rights reserved

\section{Molecular crystal global phase diagrams. III. Sufficient parameter space determination}

\author{
J. Brandon Keith ${ }^{\mathrm{a}, \mathrm{b}, \mathrm{c} *}$ and Richard B. McClurg ${ }^{\mathrm{a}, \mathrm{d}}$
}

\begin{abstract}
${ }^{a}$ Department of Chemical Engineering and Materials Science, University of Minnesota, Minneapolis, MN 55455, USA, 'bepartment of Physics and Astronomy, Brigham Young University, Provo, UT 84606, USA, 'California Institute of Technology, Division of Engineering and Applied Science, Mail Code 138-78, Pasadena, CA 91125, USA, and ${ }^{\mathbf{d}} \mathrm{SSCl}$, A Division of Aptuit, West Lafayette, IN 47906, USA. Correspondence e-mail: jbrkeith@gmail.com
\end{abstract}

In previous parts of this series [Mettes et al. (2004). Acta Cryst. A60, 621-636; McClurg \& Keith (2010). Acta Cryst. A66, 38-49] a method for constructing global phase diagrams (GPDs) for molecular crystals was developed and the method was applied to single-component ordered crystal structures of tetrahedral molecules. GPDs are useful for visualizing what types of crystal structures a given molecule may assume depending on molecular form/ interaction. Their construction uses group-theoretical methods which enumerate all possible symmetry breakings during a statistical mechanical high-to-low temperature search. In this work these results are expanded upon by outlining a method to determine a sufficiently rich parameter space to represent the experimentally observed crystal structures in a data set derived from the Cambridge Structural Database. This is significant because previous work (Mettes et al., 2004) did not specify the number of parameters needed for GPDs. Although there are suggestions in the literature that thousands of parameters are required to adequately describe tetrahedral molecule intermolecular potentials, it is found that 15 parameters are sufficient to represent the structures of the test data. The origin of this difference and its implications for determining GPD parameter values from a more detailed intermolecular potential and for interpreting GPD parameter values are discussed.

\section{Introduction}

Crystal engineering is the design and synthesis of solid-state structures with desired properties. For molecular crystals this necessitates a thorough understanding of intermolecular interactions. While the properties of isolated molecules are primarily attributable to strong covalent bonding between atoms, solid-state properties result from relatively weak interactions between molecules or low-dimensional aggregates of molecules called synthons. Two primary interactions holding together supramolecular synthons are hydrogen bonding and coordination complexation, though $\pi-\pi$, halogen-halogen and ionic interactions have also been exploited (Thalladi et al., 1996). These synthons then can be assembled into one-dimensional rods, two-dimensional sheets and three-dimensional crystal structures. Since many of the bulk properties of molecular materials are dictated by the manner in which the molecules are ordered in the solid state, crystal engineers seek to control this ordering and thus a material's electrical, optical, thermal and solubility properties (Desiraju, 1989; Braga et al., 1999; Simon \& Bassoul, 2000; Lommerse et al., 2000; Holman et al., 2001; Moulton \& Zaworotko, 2001).
Thus supramolecular chemistry depends on subtle interactions and how to control them. Non-covalent bonds have low energies and often little or no activation energy for formation. This low bond energy results in structures stabilized by difficult-to-control entropic effects, low melting points and frequent polymorphism. Likewise, as temperature changes, the balance of these effects changes, resulting in structural changes (Neumann et al., 2003). Thus energetics and thermodynamics are both essential in designing, controlling and studying molecular crystal chemistry.

Despite ongoing progress in understanding how molecular crystal structures form, there is still a need for tools to rationalize the crystal structures adopted by a molecule or collection of molecules as a function of temperature and intermolecular potential. Tools for classification and rationalization of molecular-packing tendencies under complex conditions aid crystal engineering to expand its material base and exploit new conditions to form novel structures. Phase diagrams are one such class of tools.

Global phase diagrams have been used to rationalize binary fluid mixture thermodynamics for many years. van Konyenburg \& Scott provided a general and systematic categorization of the different types of fluid-phase behavior in binary 
mixtures based upon the topology of the critical loci of a van der Waals equation of state with simple mixing rules (van Konyenburg \& Scott, 1980). They devised a set of global phase diagrams (GPDs) to classify and rationalize liquid-vapor phase behavior in terms of general non-specific molecular parameters. They showed that a simple model can predict qualitatively most of the known patterns of fluid-phase behavior, and can reveal the mechanisms of transition among the different types. Thus they describe five of the six main experimental classes of fluid behavior differentiated by the temperature-pressure projections of their critical loci. Although additional fluid-phase mixture behaviors have since been observed and a revised nomenclature is now available (Bolz et al., 1998), the original van Konyenburg system is still pervasive in the literature (Aparicio-Martinez \& Hall, 2007a,b; Cismondi \& Michelsen, 2007). Many authors have repeated this type of study in which other equations of states based on simplified models, cubic equations of state or intermolecular-potential-based equations provide the parameters of GPDs (Polishuk et al., 2000, 2002; van Pelt et al., 1995).

In a previous work we have proposed and demonstrated the construction of molecular crystal GPDs which differ from fluid-phase GPDs in fundamental ways. Gasses and liquids are both isotropic fluids which lack long-range orientational and translational order. Therefore density as a function of temperature and pressure is a sufficient order parameter for fluid phase equilibrium. Describing equilibrium among crystalline solids requires more complicated order parameters to account for the diversity of their long-range orientational and translational orderings. Also, most single-component fluids share a set of common features in their phase behaviors including a vapor/liquid equilibrium locus terminating at a critical point with universal scaling properties, a supercritical fluid region at temperatures and pressures in excess of the critical point, and an asymptotic approach toward ideal gas properties at low pressures and/or high temperatures. The exceptions to these generalizations are ionic fluids that decompose before generating a significant vapor phase. Crystalline solids lack these thermodynamic generalizations, which prevents the use of simple equation-of-state methods for generating GPDs for molecular solids.

The molecular crystal GPDs developed in this series are designed to elucidate the diverse and complex phase behavior of a set of arbitrarily shaped molecular-scale objects arranged in a long-range ordered packing rather than in disordered fluid phases. They do not use an equation of state and mixing rules but instead rely on fundamental postulates of equilibrium statistical mechanics, a set of basis functions over rotational space $S O(3)$ to represent the orientational intermolecular potential, and a set of translational packings over translational space $T^{3}$ to represent their translational ordering. The orientational interactions are the result of molecular orbitals fixed in a molecular frame of reference interacting with molecular orbitals of other molecules via the intervening orientational space $S^{2}$ so that the overall potential is a complete set over $S O(3) \times S^{2} \times S O(3)$ with intermolecular potential coefficients indexed by three angular momenta. The mathematical details are explained by Mettes et al. (2004). Representing the orientational interactions in this way leads to a countably infinite set of coefficients. A subset of these coefficients is used as the set of independent variables in GPDs. The space can then be projected into two- or three-dimensional spaces for visualization purposes. Crystalline phases occupy volumes in the GPDs and equilibrium phase transitions occur at the boundaries between the phase volumes.

The proper thermodynamic condition for equilibrium of an isothermal system is minimization of the free energy. The free energy is calculated relative to a high-temperature reference lattice in which molecules remain translationally ordered but are rotationally disordered. Experimentally this is termed a plastically crystalline state (Sherwood, 1979) and is observable for many molecules, such as adamantane or methane. Other systems melt, sublime or decompose before the plastically crystalline state is obtained. Whether or not the reference state is observed, it provides a well characterized thermodynamic reference state. This is similar to fluid phase systems which are routinely referenced to an ideal gas state, even if it has not been observed. As the temperature is lowered for a solid with fixed intermolecular potential, new phases arise through spontaneous symmetry breaking, leading to at least partial rotational ordering of the molecules.

A GPD example is shown in Figs. 1 and 2, where molecules are disordered (shown as spheres) at the center of the diagrams. As the temperature is lowered, the molecules adopt a fixed orientation. This induces a structural change from f.c.c., a high-temperature reference lattice, to one of the ten new phases shown in the figures.

A series of additional phase transitions may arise as the temperature is lowered toward absolute zero. For molecules with non-trivial point-group symmetry, it is convenient to reduce the dimensionality of the phase diagrams through adaptation of the potential to account for the molecular symmetry. This approach of expanding the angle-dependent intermolecular interactions into symmetry-adapted rotation functions was originally developed for phase transitions in heavy methane by James \& Keenan (1959) and has since been applied to crystals such as solid $\mathrm{C}_{60}$ (Michel et al., 1992; Lamoen \& Michel, 1999). Figs. 1 and 2 were constructed for molecules with tetrahedral point-group symmetry.

Like van Konyenburg's fluid-phase GPDs, molecular crystal GPDs are useful as a classification tool. They can be used in data mining intermolecular forces from structural repositories such as the Cambridge Structural Database (CSD) (Allen \& Motherwell, 2002), Crystallography Open Database (Gražulis et al., 2009) or Virtual Neutron Facility (Lin \& Keith, 2009). Also, by locating new structures that may have more desirable properties than an existing crystal structure, GPDs provide feedback for molecular synthesis for crystal engineering. Further, GPDs can provide rationalization and/or prediction of crystal polymorphs for a given molecule. Metastable polymorphs, for example, are likely to be adjacent phases on GPDs because their free energies are slightly higher than the global minimum. 


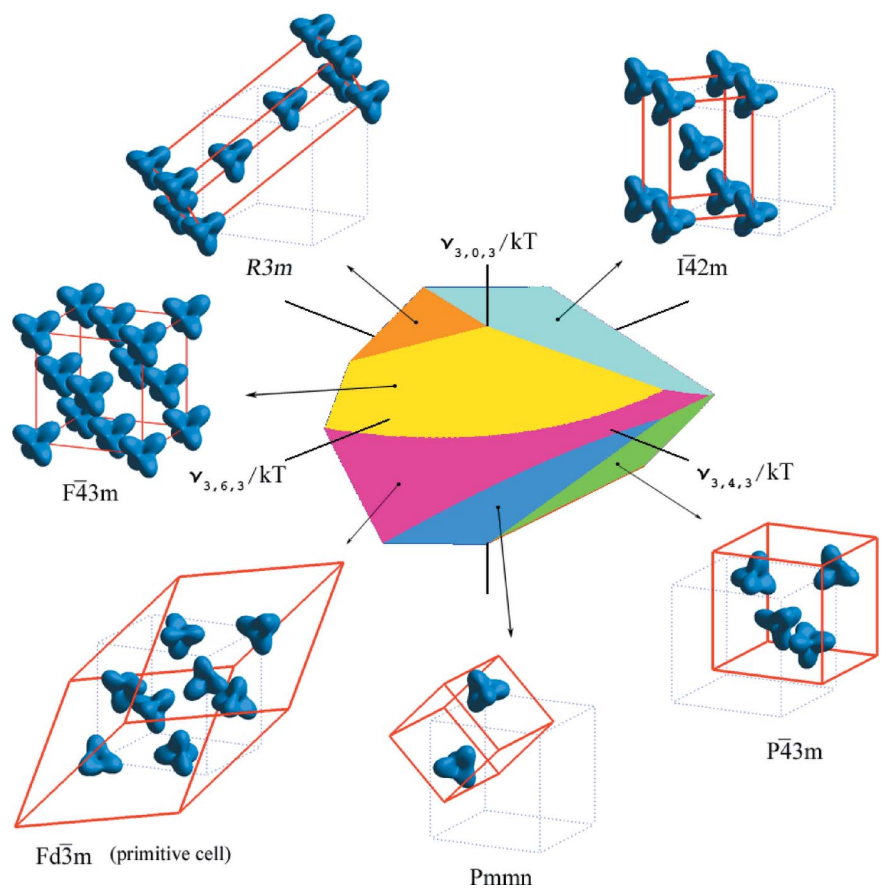

Figure 1

[111] view of a GPD for tetrahedral molecules in a face-centered cubic (f.c.c.) reference lattice. Axes are intermolecular potential coefficients $v$ scaled by $k T$. The origin of the axes is the infinite-temperature reference state. Rays protruding from the surfaces pointing at distinct phases represent ratios of potential coefficients that form the indicated space groups. Colored surfaces are boundaries between daughter phases and the reference f.c.c. phase. Reproduced with permission of the International Union of Crystallography from Mettes et al. (2004)

In a companion paper (McClurg \& Keith, 2010) we classify experimental crystal structures composed of tetrahedral point group molecules to determine what fraction of structures are amenable to inclusion in the GPDs and the number of reference lattices necessary to span the observed structures. We find that $60 \%$ of crystal structures composed of molecules with $T_{d}$ point-group symmetry are amenable and that eight reference lattices are sufficient to span the observed structures. In the remaining $40 \%$ of the structures, the molecules form synthons that pack to generate the three-dimensional crystal structure. This kind of hierarchical structure is not incorporated in the GPDs as described by Mettes et al. (2004). For the majority of structures in the test set, eight reference lattices are sufficient to describe the lattice of molecular centers of mass. We consider a set of GPDs, one for each reference lattice.

This work discusses the issue of a minimal set of intermolecular potential coefficients needed to construct GPDs. For GPDs to be useful, the observed crystal structures for an ensemble of molecules with a given point-group symmetry should occupy volumes of the GPD with a truncated set of intermolecular potential coefficients. If the full infinite parameter set is needed, then the GPDs lose much of their utility. It has been suggested that too many parameters are needed to describe intermolecular potentials for practical use in GPDs (Briels, 1980). We find that a smaller number of parameters are sufficient. This finding greatly enhances the utility of GPDs

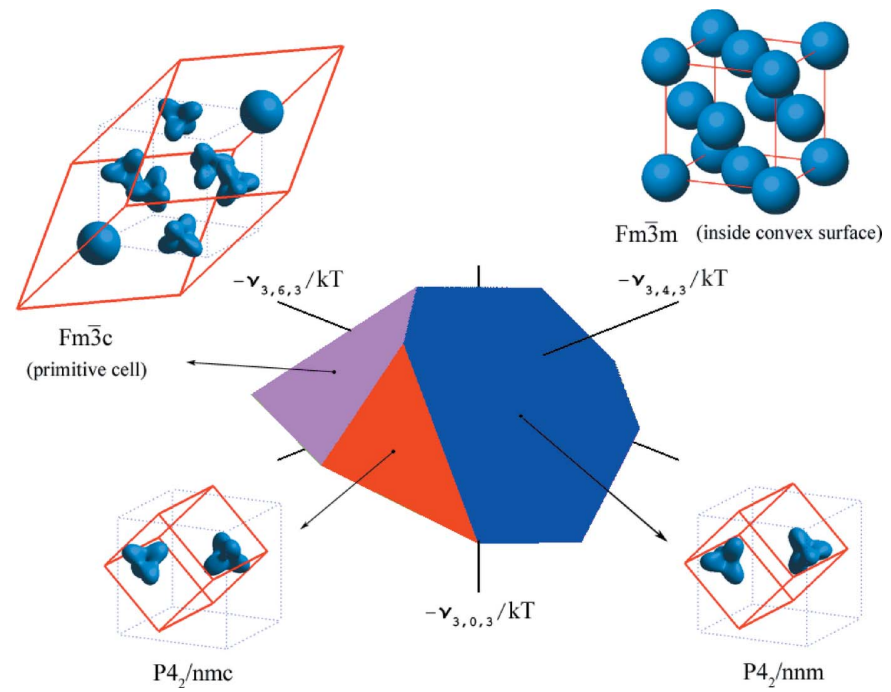

Figure 2

[111] view of a GPD for tetrahedral molecules in an f.c.c. reference lattice. Some molecules such as those in the $F m \overline{3} m$ structure appear 'spherical' because of rapid molecular reorientation. Reproduced with permission of the International Union of Crystallography from Mettes et al. (2004).

for materials design. The smaller set of parameters allows construction of manageably small-dimensional diagrams. Still, three-dimensional projections are required to produce visualizations with which users may interact and explore crystalstructure relationships and proximity in crystal phase space. Molecular crystal GPDs with a manageable number of parameters would be valuable to crystal engineers seeking to coerce an optically active molecule through synthetic perturbations to crystallize in a non-centrosymmetric space group, for example. They could also prove useful in polymorph screening to enumerate polymorphs of active pharmaceutical ingredients.

To explore the requisite number of parameters necessary to construct molecular crystal GPDs, we begin in $\$ 2$ by recalling the rotational potential used previously by Mettes et al. (2004). We then outline the computational procedure to find potential parameters for each structure in the same experimental data set used by McClurg \& Keith (2010). This is done using global optimization techniques among intermolecular potential parameter and translational symmetry space. In $\S 3$ we discuss the structures that are successfully located in potential parameter space using our candidate structure library and intermolecular potential. We also identify exceptions that cannot be located using the method utilized and make recommendations for further work to rectify these omissions.

\section{Representative potential determination}

Global phase diagrams require (i) reference lattices consistent with molecular centers of mass in experimental structures and (ii) intermolecular potential (IP) parameters to use as independent parameters. McClurg \& Keith (2010) found that the majority of the experimental structures composed of tetrahedral molecules in the CSD can be classified using only four 

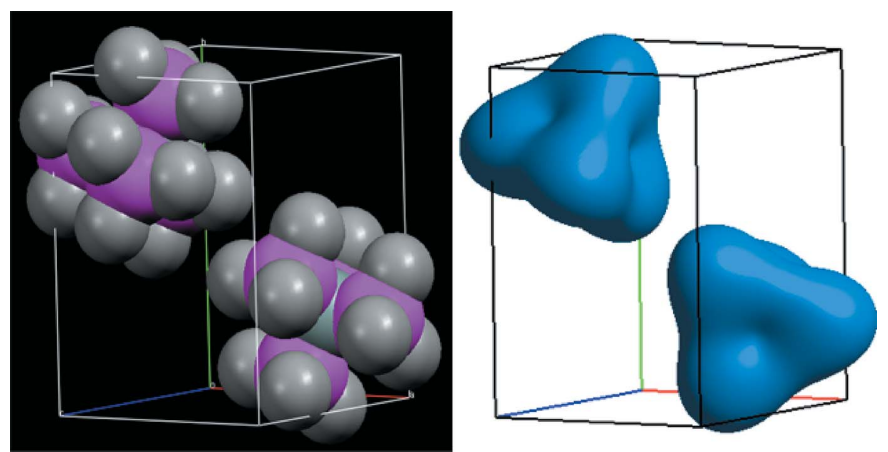

Figure 3

Comparison of an experimental structure, tetrakis(trimethylstannyl)silane [CSD structure MEZDIE01], which crystallizes in space group 2 at Wyckoff point $i$ with arbitrarily shaped tetrahedral figures whose orientation is determined by orientational energy minimization with the molecular center of mass on an ideal b.c.c. reference lattice.

reference lattices. Since there are too few examples for other reference lattices, we focus on the set \{b.c.c., f.c.c., h.c.p., s.c.\} (body-centered cubic, face-centered cubic, hexagonal close packed and simple cubic, respectively) and use these as a test set to see how many parameters are required in molecular crystal GPDs. Each of these reference lattices has at least four crystal structures with monomer synthons in the data set. This means a given structure can be categorized as a subgroup of one of these reference lattices when single molecules are reduced to spheres (as opposed to dimers or chains; see McClurg \& Keith, 2010). In this section we seek to determine a sufficient parameter space such that experimental structures are each stable in a finite volume in the IP parameter space. Thus we review our choice of IP and define the potential parameters. Then we outline a method for identifying representative potential parameters for each experimental structure. This is done using a low-temperature structural limit which is convenient and consistent with the experimental database but is not strictly necessary. Then the IP is truncated to create a finite-dimensional IP parameter space. A library of alternative crystal structures is constructed with which to compare experimental structures, and a figure of merit is specified when searching for the potential parameters. This procedure identifies a structure with similar cell shape, molecular center of mass and molecular orientations, as illustrated in Fig. 3.

\subsection{Mesoscopic Hamiltonian}

Previously (Mettes et al., 2004) we discussed the construction of a nearest-neighbor potential for molecules interacting primarily through van der Waals interactions. The potential $V$ is a level of abstraction above an atom-atom or site-site potential but retains a firm basis in quantum mechanics (van der Avoird et al., 1994). In Mettes et al. (2004) we discuss how the rotational contribution $V_{\text {or }}$ of $V$ consists of a two-center expansion constructed by coupling one-center basis functions $U_{m_{\tau} n_{\sigma}}^{\ell_{i}}$ for pairs of molecules $i$ and $j$ through a coupling 'matrix function' $J_{m_{\tau} n_{\sigma} m_{\rho} n_{\mu}}^{\ell_{i} \ell_{j}}$

$$
\begin{aligned}
V_{\text {or }}= & (1 / 2) \sum_{i j} \sum_{\ell_{i} \ell_{j} m_{\tau} m_{\rho} n_{\sigma} n_{\mu}} U_{m_{\tau} n_{\sigma}}^{\ell_{i}}\left(\boldsymbol{\omega}_{i}\right) \\
& \times J_{m_{\tau} n_{\sigma} m_{\rho} n_{\mu} \ell_{\mu}}\left(\boldsymbol{v}, \boldsymbol{\Omega}_{i j}\right) U_{m_{\rho} n_{\mu}}^{\ell_{j}}\left(\boldsymbol{\omega}_{i}\right) .
\end{aligned}
$$

The one half avoids overcounting pairwise interactions, $\ell_{i}, \ell_{j} \in \mathbb{N}$, the natural numbers, $m_{\tau, \rho} \in\left\{\ell_{i, j} \ldots-\ell_{i, j}\right\}$ and $n_{\sigma, \mu} \in\left\{\ell_{i, j} \ldots-\ell_{i, j}\right\}$. A complete derivation of equation (1) is given in Appendix $A$, but here we explore its main components.

The functions $U_{m_{\tau} n_{\sigma}}^{\ell_{i}}\left(\omega_{i}\right)$ are functions of the orientation of the molecule through its Euler angles $\omega_{i}$ using the passive convention (Varshalovich et al., 1988). They are projected from $S O(3)$ irreducible representations (IRs), also called Wigner functions, and contain both the point-group symmetry of the molecule and that of the Wyckoff point in the crystal,

$$
U_{m_{\tau} n_{\sigma}}^{\ell_{i}}\left(\omega_{i}\right)=\sum_{m_{i} n_{i}} S_{m_{i} m_{\tau}}^{\ell_{i} *} D_{m_{i} n_{i}}^{\ell_{i}}\left(\omega_{i}\right) S_{n_{i} n_{\sigma}}^{\ell_{i}} .
$$

The sparse unitary matrix $\mathbf{S}$ provides the linear combinations of Wigner functions which give a particular point-group IR symmetry (Bradley \& Cracknell, 1972). Specifically, the left multiplication by $\mathbf{S}^{-1}$ in equation (2) gives basis functions transforming like Wyckoff point-group IRs and the right multiplication by $\mathbf{S}$ gives basis functions of the molecular point-group IRs. Subscript $\tau$ is a compound index referring to multiple copies of the Wyckoff point-group IR subduced in $\ell_{i}$ and $m_{\tau}$ spans the dimensions of each IR. Subscript $\sigma$ is a compound index referring to multiple copies of the molecular point-group unit IR subduced in the $\ell_{i}$ th manifold of $S O(3)$ and $n_{\sigma}$ is its dimension. Point-group IR subduction frequencies in spherical harmonics are discussed elsewhere (Bradley \& Cracknell, 1972) and in $\$ 2.2$. The symmetry adaption leaves relatively few basis functions for molecules with non-trivial point-group symmetry since only matrix elements where $\sigma$ is the unit IR (i.e. $A_{1}$ ) are kept. This gives basis functions in the molecular frame with the full molecular symmetry. Thus the set $\left\{U_{m_{\tau} n_{\sigma}}^{\ell_{i}}\right\}$ is a complete set of basis functions taking full advantage of molecular symmetry.

The coupling matrix $J_{m_{\tau} n_{\sigma} m_{\rho} n_{\mu}}^{\ell_{i} \ell_{j}}$ specifies the angular dependence with respect to molecular centers,

$$
\begin{aligned}
J_{m_{\tau} n_{\sigma} m_{\rho} n_{\mu}}^{\ell_{i} \ell_{j}}\left(\boldsymbol{v}, \boldsymbol{\Omega}_{i j}\right)= & \sum_{\ell m_{i} m m_{j}} v_{\ell_{i}, \ell, \ell_{j}}^{n_{\sigma}, n_{\mu}}\left(r_{i j}\right)\left(\begin{array}{ccc}
\ell_{i} & \ell & \ell_{j} \\
m_{i} & m & m_{j}
\end{array}\right) \\
& \times S_{m_{\tau} m_{i}}^{\ell_{i}} C_{m}^{\ell}\left(\mathbf{\Omega}_{i j}\right) S_{m_{\rho} m_{j}}^{\ell_{j}},
\end{aligned}
$$

where $\quad \ell \in\left\{\ell_{i}+\ell_{j} \ldots\left|\ell_{i}-\ell_{j}\right|\right\}, \quad m_{i, j} \in\left\{\ell_{i, j} \ldots-\ell_{i, j}\right\} \quad$ and $m \in\{\ell \ldots-\ell\}$. The potential coefficients $v_{\ell_{i}, \ell, \ell_{j}}^{n_{\sigma}, n_{\mu}}\left(r_{i j}\right)$ are a function of the distance $r_{i j}$ between molecular centers. The neighbor distance $r_{i j}$ and orientation $\Omega_{i j}$ of the intermolecular vector are determined by the reference lattice. Since we consider pairwise interactions only among the equidistant nearest neighbors of the reference lattices, the functions $v_{\ell_{i}, \ell, \ell_{j}}^{n_{\sigma}, n_{\mu}}\left(r_{i j}\right)$ are only evaluated at the nearest-neighbor distance and are treated as scalars. Thus the coupling matrix $J_{m_{\tau} n_{\sigma} m_{\rho} n_{\mu}}$ contains reference-lattice information through $\Omega_{i j}$ and $r_{i j}$ as well as pairwise intermolecular potential information through the coefficients $\boldsymbol{v}$. These coefficients have inversion symme- 
tries that reduce the number of independent values, even for asymmetric molecules. In particular $v_{\ell_{i}, \ell, \ell_{j}}^{n_{\sigma}, n_{\mu}}$ is zero if $\ell_{i}+\ell+\ell_{j}$ is odd and $v_{\ell_{i}, \ell, \ell_{j}}^{n_{\sigma}, n_{\mu}}=(-1)^{\ell_{i}+\ell_{j}} v_{\ell_{i}, \ell, \ell_{i}}^{n_{\mu}, n_{\sigma}}$ for single-component crystals (van der Avoird et al., 1980).

As discussed previously (Mettes et al., 2004), this pairwiseadditive potential implicitly contains multimolecular manybody effects in the values of $v_{\ell_{i}, \ell, \ell_{i}}^{n_{\sigma}, n_{\mu}}\left(r_{i j}\right)$ as a mean-field contribution. Also, because equation (1) is currently limited to nearest neighbors, it is most appropriate for 'van der Waals molecules', or molecular crystals held together primarily by dispersion forces rather than ionic or strongly dipolar molecular crystals which require Ewald sums over distant neighbors. van der Waals molecules seem to be the majority of molecular crystals found in the CSD, so that this pairwise additive potential over molecules with an implicit multibody contribution is a fair parametrization and useful as we seek to

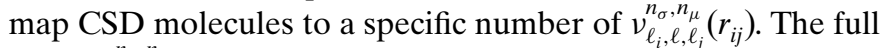
set of $v_{\ell_{i}, \ell_{\ell}}^{n_{\sigma}, n_{\mu}}\left(r_{i j}\right)$, denoted $\boldsymbol{v}$, are the axes of GPDs and constitute the search space for constructing molecular crystal GPDs.

\subsection{Computational method}

Having established translational and rotational components of the intermolecular potential as the parameter space for GPDs, we seek to determine potential parameters $v_{\ell_{i}, \ell_{\ell}, \ell_{j}}^{n_{n}, \mu_{\mu}}$ sufficient to produce each experimental crystal structure. Our method has five steps. (i) Define a figure of merit by which to order the crystal structures. (ii) Ensure the potential, equation (1), is truncated such that it geometrically is capable of producing a symmetry change from the assigned reference lattice to the experimental phase. (iii) Develop a library of structural types against which the energy of the phase of interest may be compared. (iv) Determine the energy of an experimental phase as a function of $\boldsymbol{v}$. (v) Search potential parameter space until a $\boldsymbol{v}$ vector is found which makes the experimental phase energetically minimal relative to the alternatives in the library.

2.2.1. Figure of merit. In our previous work (Keith et al., 2004; Mettes et al., 2004) linear response theory was used to seek phase transitions as a model crystal is cooled from a disordered plastic crystalline reference state at high temperature. These transitions are bifurcation points of the free-energy surface. Following each transition, the phase was identified by the presence of non-zero thermal averages of rotator functions $U_{m_{\tau} n_{\sigma}}^{\ell_{i}}\left(\omega_{i}\right)$ which serve as order parameters. This work examines the low-temperature limit of the previous model where low is relative to the temperature $T_{\mathrm{pt}}$ at which the molecules undergo the first phase transition from the reference phase. In this regime the function $U_{m_{\tau} n_{\sigma}}^{\ell_{i}}$ itself is an adequate approximation to the thermal averages of the rotator functions,

$$
\lim _{T / T_{\mathrm{pt}} \rightarrow 0}\left\langle U_{m_{\tau} n_{\sigma}}^{\ell_{i}}\right\rangle=U_{m_{\tau} n_{\sigma}}^{\ell_{i}}\left(\omega_{0}\right) .
$$

Also, the free energy $A(T)$ is equal to the potential $V$ in that limit,

$$
\lim _{T / T_{\mathrm{pt}} \rightarrow 0} A(T)=V .
$$

Equations (4) and (5) are common approximations used in many crystal-structure-prediction codes (Verwer \& Leusen, 1998) and are further justified by the exclusion of disordered structures (mesophases) in CSDSymmetry from which our data set is derived.

2.2.2. Potential truncation. The potential in equation (1) is a doubly infinite sum over manifolds $\ell_{i}$ and $\ell_{j}$ that must be truncated for practical applications. To determine an adequate truncation of the potential, we make use of space-group IRs. Symmetry-breaking mechanisms are classified by space-group IRs and an order parameter direction such as $(a, b)$ (Stokes et $a l ., 2007)$. The temperature-dependent values $a$ and $b$ are given in our model by components of space-group-IR-adapted basis functions $\mathbf{q}$ which are linear combinations of $U_{m_{\tau} n_{\sigma}}^{\ell_{i}}\left(\boldsymbol{\omega}_{i}\right)$ (Mettes et al., 2004). Space-group IR distortions of the crystal can be decomposed into point-group IR distortions of the distinguishable molecules in the crystal (Stokes et al., 1991). To determine which IRs $\tau_{w}$ of point group $w$ are in a symmetrybreaking space-group IR $\tau_{\mathrm{SG}}$, we calculate their subduction frequencies $n_{\mathrm{SG}}$,

$$
n_{\mathrm{SG}}=\frac{1}{|w|} \sum_{g \in w} \chi^{\tau_{\mathrm{SG}}}(g)^{*} \chi^{\tau_{w}}(g) \quad \forall \tau_{\mathrm{SG}},
$$

where $|w|$ is the number of elements $g$ in $w \cdot \chi(g)^{\tau_{\mathrm{SG}}}$ and $\chi(g)^{\tau_{w}}$ are the traces of the matrix representation of the space-group IR and point-group IR, respectively. The calculations are easily performed using the ISOTROPY software package (Stokes et al., 2007) using the 'show frequency' command. Space-group IR and point-group IR characters may also be generated.

Using these point groups one may also calculate the number of times each point-group IR $\tau_{w}$ appears for each manifold of $S O(3)$ or $\ell_{i}$ value,

$$
n_{S O(3)}=\frac{1}{|w|} \sum_{g \in w} \chi^{\ell_{i}}(g)^{*} \chi^{\tau_{w}}(g) \quad \forall \ell_{i},
$$

where $\chi^{\ell_{i}}$ is the trace of the matrix representation of an IR of $S O(3)$, also called a Wigner rotation matrix. Such subduction frequencies may be easily calculated but are also tabulated in standard references (Bradley \& Cracknell, 1972) and are shown for $O_{h}$ and $D_{3 h}$ in Table 1. As there is no molecular unit IR in the first, second or fifth manifold, there are no Wyckoff IR rows of $U_{m_{\tau} n_{\sigma}}^{\ell_{i}}$ so these manifolds are not shown.

If the potential is truncated before the manifold at which a Wyckoff IR forming a symmetry-breaking space-group IR is present [i.e. if $n_{\mathrm{SG}}=0$ in equation (6)], the desired phase transition cannot occur with that potential. Therefore we calculate minimal $S O(3)$ manifolds necessary to achieve a transition from the assigned reference lattice to an experimental structure. This is shown for the f.c.c. reference lattice in Table 2, where we use the convention of appending the occupied Wyckoff positions of a crystal structure to its space group (thus a structure with space group 64 in which molecular centers of mass occupy Wyckoff points $d$ and $f$ would be 
Table 1

Presence of $O_{h}$ and $D_{3 h}$ point-group IRs for various manifolds of $S O(3)$.

$O_{h}$ is the Wyckoff point group of the f.c.c., b.c.c. and s.c. reference lattices. $D_{3 h}$ is the Wyckoff point group of the h.c.p. reference lattice. The first occurrence of a given IR is shown in bold.

\begin{tabular}{lll}
\hline$\ell_{i}$ & $O_{h}$ IRs & $D_{3 h}$ IRs \\
\hline 0 & $\mathbf{A}_{1 g}$ & $\mathbf{A}_{1}^{\prime}$ \\
3 & $\mathbf{A}_{2 u}+\mathbf{T}_{1 u}+\mathbf{T}_{2 u}$ & $A_{1}^{\prime}+\mathbf{A}_{2}^{\prime}+\mathbf{E}^{\prime}+\mathbf{E}^{\prime \prime}$ \\
4 & $A_{1 g}+\mathbf{E}_{g}+\mathbf{T}_{1 g}+\mathbf{T}_{2 g}$ & $A_{1}^{\prime}+\mathbf{A}_{1}^{\prime \prime}+\mathbf{A}_{2}^{\prime \prime}+2 E^{\prime}+E^{\prime \prime}$ \\
6 & $A_{1 g}+\mathbf{A}_{2 g}+E_{g}+T_{1 g}+2 T_{2 g}$ & $2 A_{1}^{\prime}+A_{1}^{\prime \prime}+A_{2}^{\prime}+A_{2}^{\prime \prime}+2 E^{\prime}+2 E^{\prime \prime}$ \\
7 & $A_{2 u}+\mathbf{E}_{u}+2 T_{1 u}+2 T_{2 u}$ & $A_{1}^{\prime}+A_{1}^{\prime \prime}+A_{2}^{\prime}+2 A_{2}^{\prime \prime}+3 E^{\prime}+2 E^{\prime \prime}$ \\
8 & $A_{1 g}+2 E_{g}+2 T_{1 g}+2 T_{2 g}$ & \\
9 & $\mathbf{A}_{1 u}+A_{2 u}+E_{u}+3 T_{1 u}+2 T_{2 u}$ & \\
\hline
\end{tabular}

uncommon and higher-manifold-induced phase transitions are not generally observed (Lynden-Bell \& Michel, 1994). The reasons for this are different for small and large molecules. For small molecules with only a few atoms (i.e. $\mathrm{CH}_{4}$ ), molecular orbitals tend to form low-energy slowly varying topologies to lessen kinetic energy contributions in Schrödinger's equation. The result is an IP well represented by smooth slowly varying basis functions. For large molecules, low-manifold contributions to the potential are sufficient to locate attractive/repulsive regions of the potential and the relative magnitudes of attractive configurations, even though these basis functions are not sufficient to represent the fine structure in the potential due to individual atoms (Hloucha et al., 2001). In denoted $64 d, f$ ). Point-group IRs subduced in the space-group IR and the first manifold or $\ell_{i}$ value of $S O(3)$ at which a given Wyckoff IR appears are also shown. The pathways shown are calculated in ISOTROPY by finding a group-subgroup transition from the high-temperature reference phase, such as f.c.c., to the observed structure.

For some pathways, such as that of carbon tetraiodide, $225 a \rightarrow 121 a$ in Table 2, there is a single space-group IR $\Gamma_{5}^{-}$ subducing a single point-group IR $T_{2 u}$. Table 1 shows that this point-group IR first occurs in the third manifold of $S O(3)$ when split by an $O_{h}$ point group crystal field. Thus $\ell_{i}^{\max } \geq 3$ may give the $121 a$ structure but $\ell_{i}^{\max }<3$ is insufficient. For other pathways, such as that of tetrakis $\left(M_{3}\right.$-t-butylimido)tetraiodotetraindium, 225a $\rightarrow 12 i$, there is more than one Wyckoff IR subduced in a space-group IR. $E_{u}, T_{1 u}$ and $T_{2 u}$ can lead to this transition. As two of them are present at the third manifold (Table 1), a potential with $\ell_{i}^{\max } \geq 3$ may also produce this transition. For still others, such as that of adamantane, $225 a \rightarrow 114 a$, the pathway is coupled, or composed of the direct sum of two space-group IRs. Three different coupled space-group IRs decompose into three different point-group IRs. One of these pathways uses IRs on the third manifold of $S O(3)\left(\ell_{i}^{\max } \geq 3\right)$ while the other two require fourth manifold basis functions. A similar analysis for the b.c.c., h.c.p. and s.c. reference lattices given in Appendix $A$ shows that symmetrybreaking Wyckoff IRs appear by at least the third or fourth manifold in nearly all cases.

In real phase transitions, IRs on the first allowed non-trivial manifold typically induce symmetry breaking. Secondmanifold-induced symmetry breaking is
Table 2

Group-theoretical symmetry-breaking pathways of experimental lattices for the f.c.c. reference lattice.

Classified by space-group IR and order-parameter direction, each pathway shows the point-group IR and minimal manifold of $S O(3)$ in equation (1) required to achieve it. The order-parameter directions are given in an abbreviated form in the notation of Stokes \& Hatch (2002). The pathways, space-group IRs, order-parameter directions and point-group IRs were computed using ISOTROPY. However, transitions belonging to a coupled IR between a high-symmetry point and line are currently not a feature of ISOTROPY. These entries have been marked with an asterisk.

\begin{tabular}{|c|c|c|c|c|}
\hline Pathway & Space-group IR & $\begin{array}{l}\text { Order- } \\
\text { parameter } \\
\text { direction }\end{array}$ & Point-group IR & $\ell^{\text {req'd }}$ \\
\hline $225 a \rightarrow 121 a$ & $\Gamma_{5}^{-}$ & P1 & $T_{2 u}$ & 3 \\
\hline $225 a \rightarrow 142 a$ & $W_{3}$ & $\mathrm{P} 2$ & $T_{1 g}, A_{2 u}, E_{u}$ & 3 \\
\hline \multirow{3}{*}{$225 a \rightarrow 114 a$} & $X_{2}^{-} \oplus \Gamma_{5}^{-}$ & $\mathrm{P} 1 \oplus \mathrm{P} 1$ & $A_{2 u}, E_{u} \oplus T_{2 u}$ & 3 \\
\hline & $X_{3}^{+} \oplus \Gamma_{5}^{-}$ & $\mathrm{P} 1 \oplus \mathrm{P} 1$ & $T_{1 g} \oplus T_{2 u}$ & 4 \\
\hline & $X_{2}^{-} \oplus X_{3}^{+}$ & $\mathrm{P} 1 \oplus \mathrm{P} 1$ & $A_{2 u}, E_{u} \oplus T_{1 g}$ & 4 \\
\hline $225 a \rightarrow 152 b$ & $\Lambda_{3}(k=1 / 3)$ & $\mathrm{P} 1$ & $E_{g}, T_{1 g}, T_{2 g}, E_{u}, T_{1 u}, T_{2 u}$ & 3 \\
\hline $\begin{array}{l}225 a \rightarrow 15 e \\
\text { (REKYUB) }\end{array}$ & $L_{3}^{-}$ & P7 & $E_{u}^{\delta}, T_{1 u}, T_{2 u}$ & 3 \\
\hline $\begin{aligned} 225 a & \rightarrow 12 i \\
225 a & \rightarrow 64 d, f\end{aligned}$ & $\begin{array}{l}L_{3}^{-} \\
*\end{array}$ & $\mathrm{P} 2$ & $E_{u}, T_{1 u}, T_{2 u}$ & 3 \\
\hline \multirow{24}{*}{$\begin{array}{l}225 a \rightarrow 14 e \\
\quad(\text { MECKUA) }\end{array}$} & $\Gamma_{3}^{+} \oplus L_{3}^{-}$ & $\mathrm{P} 1 \oplus \mathrm{C} 8$ & $E_{g} \oplus E_{u}, T_{1 u}, T_{2 u}$ & 4 \\
\hline & $\Gamma_{3}^{+} \oplus X_{5}^{-}$ & $\mathrm{C} 1 \oplus \mathrm{S} 7$ & $E_{g} \oplus T_{1 u}, T_{2 u}$ & 4 \\
\hline & $\Gamma_{4}^{+} \oplus X_{5}^{-}$ & $\begin{array}{l}\mathrm{P} 1 \oplus \mathrm{C} 11 \\
\mathrm{P} 1 \oplus \mathrm{S} 7\end{array}$ & $T_{1 g}^{\delta} \oplus T_{1 u}, T_{2 u}$ & 4 \\
\hline & $\Gamma_{5}^{+} \oplus L_{3}^{-}$ & $\mathrm{C} 2 \oplus \mathrm{C} 8$ & $T_{2 g} \oplus E_{u}, T_{1 u}, T_{2 u}$ & 4 \\
\hline & $\Gamma_{5}^{+} \oplus X_{5}^{-}$ & $\begin{array}{l}\mathrm{P} 1 \oplus \mathrm{C} 11 \\
\mathrm{P} 1 \oplus \mathrm{S} 7\end{array}$ & $T_{2 g} \oplus T_{1 u}, T_{2 u}$ & 4 \\
\hline & $L_{2}^{+} \oplus L_{1}^{-}$ & $\mathrm{P} 1 \oplus \mathrm{P} 1$ & $A_{2 g}, T_{1 g} \oplus A_{1 u}, T_{2 u}$ & 4 \\
\hline & $L_{2}^{+} \oplus L_{3}^{-}$ & $\mathrm{P} 1 \oplus \mathrm{P} 7$ & $A_{2 g}, T_{1 g}^{\circ} \oplus E_{u}, T_{1 u}, T_{2 u}$ & 4 \\
\hline & $L_{2}^{+} \oplus X_{2}^{-}$ & $\mathrm{P} 1 \oplus \mathrm{P} 1$ & $A_{2 g}, T_{1 g} \oplus A_{2 u}, E_{u}$ & 4 \\
\hline & $L_{2}^{+} \oplus X_{3}^{-}$ & $\mathrm{P} 1 \oplus \mathrm{P} 1$ & $A_{2 g}, T_{1 g} \oplus E_{u}, T_{1 u}$ & 4 \\
\hline & $L_{2}^{+} \oplus X_{5}^{-}$ & $\mathrm{P} 1 \oplus \mathrm{P} 1$ & $A_{2 g}, T_{1 g} \oplus T_{1 u}, T_{2 u}$ & 4 \\
\hline & $L_{3}^{+} \oplus L_{1}^{-}$ & $\mathrm{P} 7 \oplus \mathrm{P} 1$ & $E_{g}, T_{1 g}, T_{2 g} \oplus A_{1 u}, T_{2 u}$ & 4 \\
\hline & $L_{3}^{+} \oplus L_{3}^{-}$ & $\mathrm{P} 7 \oplus \mathrm{P} 7$ & $E_{g}, T_{1 g}, T_{2 g} \oplus E_{u}, T_{1 u}, T_{2 u}$ & 4 \\
\hline & $L_{3}^{+} \oplus X_{2}^{-}$ & $\mathrm{P} 7 \oplus \mathrm{P} 1$ & $E_{g}, T_{1 g}, T_{2 g} \oplus A_{2 u}, E_{u}$ & 4 \\
\hline & $L_{3}^{+} \oplus X_{3}^{-}$ & $\mathrm{P} 7 \oplus \mathrm{P} 1$ & $E_{g}, T_{1 g}, T_{2 g}^{\circ} \oplus E_{u}, T_{1 u}$ & 4 \\
\hline & $L_{3}^{+} \oplus X_{5}^{-}$ & $\mathrm{P} 7 \oplus \mathrm{P} 1$ & $E_{g}, T_{1 g}, T_{2 g} \oplus T_{1 u}, T_{2 u}$ & 4 \\
\hline & $L_{1}^{-} \oplus L_{3}^{-}$ & $\mathrm{P} 1 \oplus \mathrm{C} 8$ & $A_{1 u}, T_{2 u} \oplus E_{u}, T_{1 u}, T_{2 u}$ & 4 \\
\hline & $L_{1}^{-} \oplus X_{2}^{-}$ & $\mathrm{P} 1 \oplus \mathrm{P} 1$ & $A_{1 u}, T_{2 u} \oplus A_{2 u}, E_{u}$ & 4 \\
\hline & $L_{1}^{-} \oplus X_{3}^{-}$ & $\mathrm{P} 1 \oplus \mathrm{P} 1$ & $A_{1 u}, T_{2 u} \oplus E_{u}, T_{1 u}$ & 4 \\
\hline & $L_{1}^{-} \oplus X_{5}^{-}$ & $\mathrm{P} 1 \oplus \mathrm{P} 1$ & $A_{1 u}, T_{2 u} \oplus T_{1 u}, T_{2 u}$ & 4 \\
\hline & $L_{3}^{-} \oplus X_{2}^{-}$ & $\mathrm{P} 7 \oplus \mathrm{P} 1$ & $E_{u}, T_{1 u}, T_{2 u} \oplus A_{2 u}, E_{u}$ & 4 \\
\hline & $L_{3}^{-} \oplus X_{3}^{-}$ & $\mathrm{P} 7 \oplus \mathrm{P} 1$ & $E_{u}, T_{1 u}, T_{2 u} \oplus E_{u}, T_{1 u}$ & 4 \\
\hline & $L_{3}^{-} \oplus X_{5}^{-}$ & $\mathrm{P} 7 \oplus \mathrm{P} 1$ & $E_{u}, T_{1 u}, T_{2 u} \oplus T_{1 u}, T_{2 u}$ & 4 \\
\hline & $X_{2}^{-} \oplus X_{5}^{-}$ & $\begin{array}{l}\mathrm{P} 1 \oplus \mathrm{C} 11 \\
\mathrm{P} 1 \oplus \mathrm{S} 7\end{array}$ & $A_{2 u}, E_{u} \oplus T_{1 u}, T_{2 u}$ & 4 \\
\hline & $X_{3}^{-} \oplus X_{5}^{-}$ & $\mathrm{P} 1 \oplus \mathrm{C} 11$ & $E_{u}, T_{1 u} \oplus T_{1 u}, T_{2 u}$ & 4 \\
\hline $\begin{array}{l}225 a \rightarrow 14 e \\
\quad(\text { TOHSUE) }\end{array}$ & $\Delta_{5}(k=1 / 4)$ & $\mathrm{C} 7$ & $T_{1 g}, T_{2 g}, T_{1 u}, T_{2 u}$ & 3 \\
\hline $225 a \rightarrow 15 f, f, f, f$ & $C_{2}\left(k_{1}=1 / 4, k_{2}=3 / 4\right)$ & C18, C19 & $\begin{array}{l}A_{2 g}, E_{g}, T_{1 g}, T_{2 g}, \\
A_{1 u}, E_{u}, T_{1 u}, T_{2 u}\end{array}$ & 3 \\
\hline
\end{tabular}


either case, the most important aspects of the IP are given by slowly varying functions while more rapidly varying functions produce finer details. Thus as a minimal basis set we truncate equation (1) at $\ell_{\max }=3$ or 4 .

Truncating at $\ell_{\max }=4$ leaves 15 coefficients in the potential, $v_{0,3,3}, v_{0,4,4}, v_{3,0,3}, v_{3,2,3}, v_{3,4,3}, v_{3,6,3}, v_{3,1,4}, v_{3,3,4}, v_{3,5,4}, v_{3,7,4}, v_{4,0,4}$, $v_{4,2,4}, v_{4,4,4}, v_{4,6,4}$ and $v_{4,8,4}$. The coefficient $v_{0,0,0}$ is negligible since it only affects the trivial basis function $U_{0,0}^{0}=1$ which is isotropic and therefore unimportant in rotational ordering. As the unit IR for $T_{d}$ appears once in the zeroth, third and fourth manifolds, $n_{\sigma}$ and $n_{\mu}$ are always $1_{A_{1}}$ and have been dropped in the notation for the coefficients in equation (3). For space groups whose occupied Wyckoff point group in the reference lattice has the inversion as one of its symmetry operators, $v_{0, x, x}$ cancels in the crystal field for odd values of $x$. This is the case with the $v_{033}$ coefficient in the b.c.c., f.c.c. and s.c. reference lattices with 14 coefficients but not for h.c.p. with 15 .

2.2.3. Candidate lattice library. We seek a set of potential parameters $v$ such that the energy of an observed structure is lower than other alternatives, whether the alternative structures are experimentally observed or not. In our previous work (Mettes et al., 2004), high-symmetry point IR distortions (Stokes \& Hatch, 1988; Stokes et al., 2007) from a reference lattice led to structures classified using their isotropy subgroups (ISs). These subgroups represent the most common types of phase transitions and are minimal in the sense that only one domain of each structure is tested. This compares favorably with many crystal-structure-prediction algorithms which generate thousands of multi-domain duplicate structures (Gavezzotti, 2007). Therefore they are convenient for constructing a library of candidate lattices. They are discussed further in $\$ 3$. As most molecular crystals have one molecule or less per asymmetric part of the unit cell $\left(Z^{\prime} \leq 1\right)$, we have discarded ISs that imply more than one occupied Wyckoff point (Padmaja et al., 1990). ISs whose primitive unit cell contains more than eight molecules are also discarded (Gdanitz, 1997). We note that these constraints are made based on what is commonly observed in the CSD and can be incrementally extended in order to achieve a larger candidate library.

To minimize the energy of these candidate lattices, basis functions $U_{m_{\tau} n_{\sigma}}^{\ell_{i}}\left(\omega_{i}\right)$ are placed at one molecular center of the molecular positions of the lattice of each IS and space-group operations of the IS are applied to generate basis functions $U_{m_{\tau} n_{\sigma}}^{\ell_{i}}\left(\omega_{i}\right)$ for all other molecules in the Wyckoff orbit. Although these candidate lattices are generated from ISs, after Euler angle minimization they are free to assume whatever structure the minimization algorithm can find provided the reference orientations of the basis functions $U_{m_{\tau} n_{\sigma}}^{\ell_{i}}\left(\omega_{i}\right)$ are related by IS space-group operations. Thus a candidate lattice has fixed (super-) cell parameters and Wyckoff points but variable Euler angles and space group. This procedure gives a candidate lattice library that, at a minimum, includes all highsymmetry $k$-point isotropy subgroups but which can contain other lower-symmetry structures as well [owing to additional symmetry breaking while rotating the basis functions $\left.U_{m_{\tau} n_{\sigma}}^{\ell_{i}}\left(\omega_{i}\right)\right]$ and provided translational symmetry requirements are met. As indicated above, this candidate library may be systematically improved by explicitly including new isotropy subgroup supercells.

Applying this procedure yielded 75 candidate lattices for b.c.c., 67 for f.c.c., 19 for h.c.p. and 105 for s.c. Minimizing the energy of all candidate lattices gives a lowest-energy structure with which to compare the energy of the experimental structure while searching parameter space. Although these candidate lists are remarkably small compared with the thousands of structures generated in many crystal-structure-prediction programs, they are sufficient to place most of the observed structures as discussed in $\S 3$.

2.2.4. Experimental structure energies. With a set of candidate lattices with which to compare a given observed structure in $\boldsymbol{v}$ space, we now need to find the energy of each observed structure, leaving $\boldsymbol{v}$ unspecified. This can be done by translating the atomic positions of the observed structure into the needed Euler angles $\omega$ and intermolecular angles $\Omega$ of equation (1). The intermolecular angles $\Omega$ are easy to obtain because they are determined by the reference lattice, but the Euler angles are dependent upon the orientation of the molecules in the reference lattice. This is complicated by the fact that the experimental atomic positions in each molecule are slightly distorted from perfect molecular $T_{d}$ point-group symmetry and are slightly shifted from the ideal Wyckoff points of their reference lattice. This is due to small strains resulting from embedding the experimental lattice in the almost perfect but nonetheless approximate symmetry of its reference lattice (see McClurg \& Keith, 2010). To obviate these difficulties, the reference-lattice molecular centers of mass are first slightly shifted to coincide with the centers of mass of the molecules in their native space group. Then the Euler angles are calculated by rotating a rigid tetrahedral molecule to minimize its atomic distances compared with the coordinates of the experimental molecule. Thus the Euler angles of a perfect tetrahedron are obtained by a 'best fit' to molecular coordinates of a slightly distorted tetrahedron. We note the reference orientation of the molecule is with three-

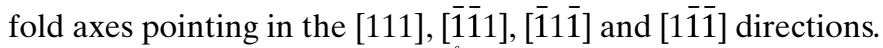

Next the rotator functions $U_{m_{\tau} n_{\sigma}}^{\ell_{i}}$ may be calculated directly using equation (2). $U_{m_{\tau} n_{\sigma}}^{\ell_{i}}$ is fully symmetric with respect to molecular point-group symmetry and is not influenced by nonunique Euler angles. Substituting $U_{m_{\tau} n_{\sigma}}^{\ell_{i}}$ into equation (1) yields the interaction potential as a function of the potential parameters $\boldsymbol{v}$. This gives the energy $E=V(\boldsymbol{v})$ as a linear polynomial in $\boldsymbol{v}$. For instance,

$$
\begin{aligned}
E= & 0.2901 v_{0,4,4}-0.0028 v_{3,0,3}+0.0036 v_{3,1,4} \\
& -0.1020 v_{3,2,3}-0.0425 v_{3,3,4}-0.0127 v_{3,4,3} \\
& +0.0053 v_{3,5,4}-0.0006 v_{3,6,3}+0.0071 v_{3,7,4} \\
& -0.0055 v_{4,6,4}-0.0183 v_{4,8,4}+1.2640 v_{4,0,4} \\
& +0.0109 v_{4,2,4}+0.0316 v_{4,4,4},
\end{aligned}
$$

for tetrakis(trimethylstannyl)silane [CSD structure MEZDIE01], which crystallizes in space group 2.

2.2.5. Minimum energy gap. Now that we have a potential that is capable of producing all the experimental structures 
from McClurg \& Keith (2010) for the \{f.c.c., s.c., h.c.p., b.c.c.\} reference lattices, and we have input each structure's molecular Euler angles to reduce it to a form such as equation (8), we need to develop an algorithm that will isolate this structure in $v$ space as the most thermodynamically stable structure. To this end we propose a general minimum energy difference algorithm that makes use of the candidate lattice libraries derived in $\$ 2.2 .3$.

This algorithm proceeds as follows. First, normalize the $v$ vector by projecting it onto the unit hypersphere, since only relative $\boldsymbol{v}$ magnitudes affect the phase as $T \rightarrow 0$. Then, taking the library of structures discussed previously in this section, minimize the difference of the energy of the target structure $E_{\text {target }}$ and the minimal energy structure in the collection $\left\{E_{\mathrm{lib}}(\boldsymbol{v}, \boldsymbol{\omega})\right\}$. Thus a vector $\nu_{\mathrm{RP}}$ is sought that minimizes

$$
\Delta E=E_{\text {target }}(v)-\min \left\{E_{\text {lib }}(\boldsymbol{v}, \boldsymbol{\omega})\right\} .
$$

We note that, for a finite library, this gives the point in $\boldsymbol{v}$ space of the largest energy gap between $E_{\text {target }}$ and any other structures in the library and is representative of the family of intermolecular potentials consistent with the target structure. For this reason it is termed the representative potential (RP); it is characterized by the representative parameter vector $v_{\mathrm{RP}}$. For an infinite library, $\Delta E$ is bounded below by zero since $\left\{E_{\mathrm{lib}}(\boldsymbol{v}, \boldsymbol{\omega})\right\}$ contains the target structure and is minimized with respect to $\omega$ while the target structure is held at fixed molecular orientation.

While $E_{\text {target }}$ is based on observed Euler angles $\omega$ and is only a function of $\boldsymbol{v}$ [i.e. equation (8)], $E_{\text {lib }}$ contains a library of structures whose energies are functions of $\boldsymbol{v}$ and $\boldsymbol{\omega}$. Since minimizing with respect to $\omega$ is computationally demanding, we solve equation (9) in an iterative manner. First equation (9) is minimized for each experimental lattice with $\left\{E_{\text {lib }}\right\}$ based only on the energies of the remaining experimental lattices. For each trial $\boldsymbol{v}_{\mathrm{RP}}$ found from this relative minimization, all additional candidate lattices are minimized with respect to their Euler angles at fixed $\boldsymbol{v}$ and the lowest-energy solution is appended to the library. Again equation (9) is minimized for the energy of each experimental structure with respect to the augmented set to find a new trial RP in $v$ space and the process iterates until the RPs converge.

Minimization methods using genetic algorithms (Holland, 1992) (differential evolution) and simulated annealing (Kirkpatrick et al., 1983) are used on the Euler angle minimization and energy-difference minimization, respectively. This is particularly important since the topologies of the energy and energy difference are both non-linear with many local minima, the latter also having discontinuous derivatives. We reiterate that this is a relative energy minimization for $\Delta E$ in parameter space $\boldsymbol{v}$ and not a simulated annealing with fixed potential and varying temperature, as is more commonly done.

Since the goal of our work is to determine the minimum truncation point that provides a sufficiently rich potential to span the observed crystal structures, we adopt an iterative procedure that begins with lower-order manifolds and proceeds to higher orders if necessary. First, the energy difference is minimized using $\ell_{\max }=3$ for all experimental structures. Only if a minimum is not found for a given structure are fourth manifold basis functions appended to the parameter space and the process repeated.

\section{Discussion and conclusion}

\subsection{Representative potentials}

Calculating potential parameters according to the procedure outlined in $\$ 2$ gives the results in Table 3 where the potential coefficients are given for experimental structures in the four most prevalent reference lattices. Calculations for other reference lattices such as the distorted diamond cubic reference lattices identified in part II of this series (McClurg \& Keith, 2010) are not reported here since they represent a smaller percentage of distinct structures. The difference in energy between the experimental structure and the nextlowest-energy structure among the candidate lattices is shown in the second column.

For eight of the 35 structures, $\Delta E<0$. This indicates that the target structure is not directly in the library but has been bounded in potential parameter space. Thus $\Delta E=0$ on the borders, but $\Delta E<0$ in the interior. $v_{\mathrm{RP}}$ gives a set of potential parameters where the target structure is relatively stable and is expected to be near the center of the region.

For 18 of the 35 structures, $\Delta E=0$. This indicates the target structure is in the library and $\omega_{\text {exp }} \simeq \omega_{\min }$. Since $\Delta E=0$ throughout the region where the target is stable, $v_{\mathrm{RP}}$ is an arbitrary point in the region and is not necessarily centrally located.

For six of the 35 structures, $0<\Delta E<0.01$, which is close enough to zero to conclude that a representative potential for these structures has been found. This can be asserted because the potential is composed of products of $U_{m_{\tau} n_{\sigma}}^{\ell_{i}}\left(\omega_{i}\right)$, ClebschGordan coefficients, spherical harmonics and unit sphere normalized potential coefficients $\boldsymbol{v}$ that are all unitary. This makes the energy and therefore energy difference to be $\mathcal{O}(1)$. An energy difference less than this tolerance likely results from the method employed to determine orientational Euler angles for the experimental structures, $\omega_{\text {exp }}$, and/or incomplete minimization in the determination of Euler angles at the global minimum, $\omega_{\min }$. Whereas $\omega_{\text {exp }}$ is determined by analysis of the experimental structures, which is subject to small errors, the minimizer is free to vary $\omega_{\min }$ to attain the lowest possible energy. Thus the small positive energy differences in these cases are attributed to inaccuracies in the parameters used to describe the experimental structures and $\boldsymbol{v}_{\mathrm{RP}}$ is therefore likely to be in the correct region of parameter space. A close approximation to the experimental structure is contained in the comparison library, and therefore the RP may not be centrally located in its domain for these cases for the same reasons as discussed above for the $\Delta E=0$ set.

For three of the 35 structures, $\Delta E>0.01$. This indicates the absence of potential parameters consistent with the experimental crystal structure relative to the other structures using the truncation specified. The $\boldsymbol{v}_{\mathrm{RP}}$ listed identifies a point in 
Table 3

RP components for crystals of tetrahedral molecules in the CSD.

The 'Identifier' is a representative structure for a given structural type. The 'Multiplicity' is the number of such structures in our data set. The parameters $v_{\ell_{i}, \ell, \ell_{j}}$ are indexed according to equation (1) and have been mapped to the unit hypersphere.

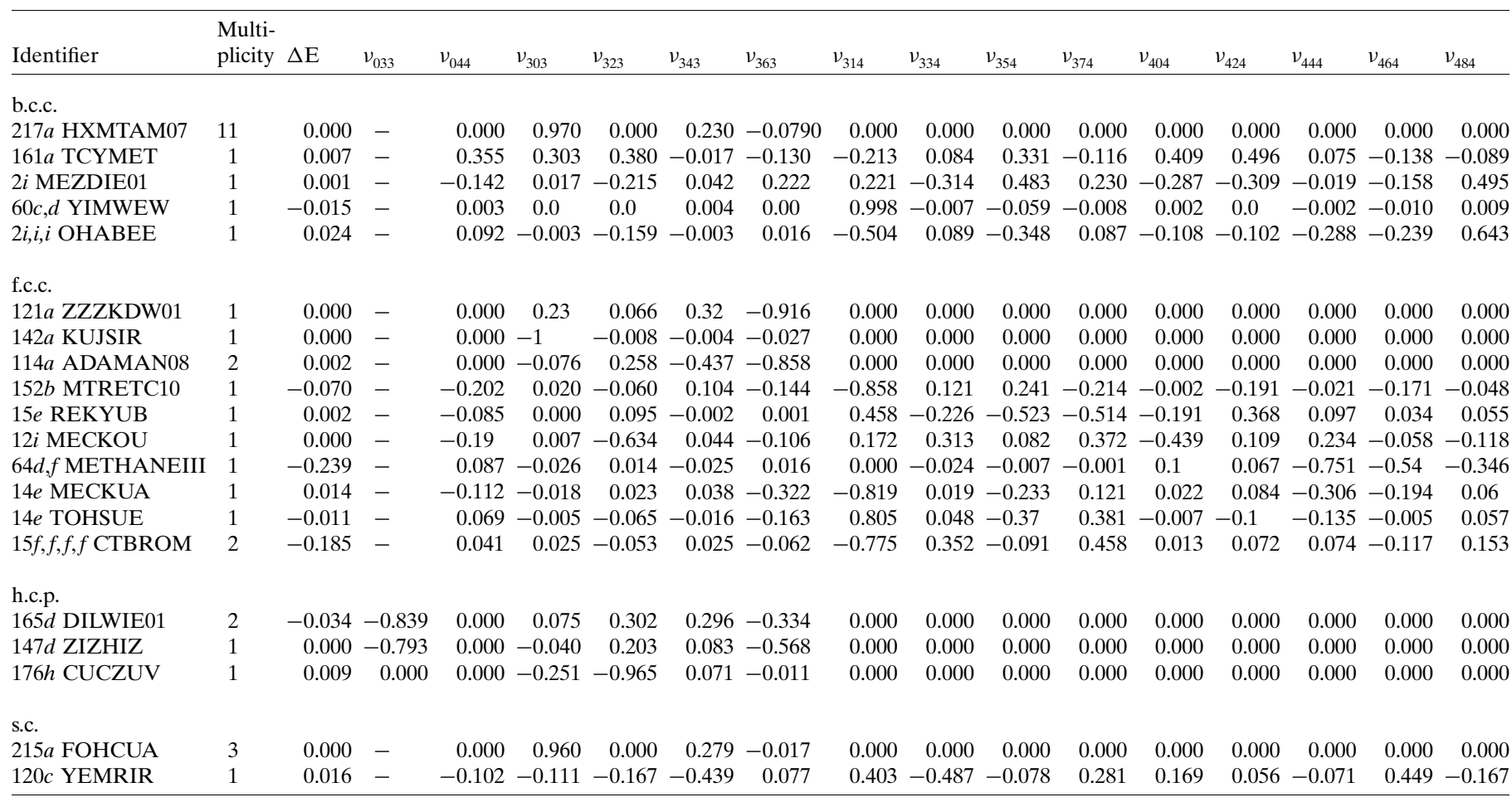

parameter space where the target structure is least metastable compared with the global minimum in the library. The target structure is also usually quite similar to the global minimal structure. This is the case with YEMRIR, for example. It has a positive $\Delta E$ but is similar to the global minimum structure, space group 132 with molecules at Wyckoff point $a$. Both have two molecules per unit cell and one of the molecules, that at $(0,0,0)$ in both structures, has the same orientation. The difference is the second molecule which is rotated $\sim 90^{\circ}$ between the two structures. Reasons for this difference may be related to truncation of the potential after the fourth manifold, lack of molecular displacements and lattice strains in the model, lack of explicit second-nearest-neighbor interactions, the low-temperature limit discussed in \$2.2.1, or missing quantum effects. Another reason may be that the reported experimental phase is in fact metastable in the limit $T \rightarrow 0$. Ostwald's step rule supports this possibility. The rule is based on the observation that during crystal nucleation and growth a metastable phase frequently crystallizes first which is closest in structure to the liquid or solution from which it is grown and which has the lowest free-energy barrier. The crystallite then undergoes a series of phase changes until it reaches the thermodynamically stable structure (Schmalzried, 2003). The experimentally observed structure may have formed during an early stage of nucleation as one of these intermediate structures. No attempts have been made to attribute either of these two outlying cases to one or more of the above plausible explanations.
Twenty of the 35 distinct structures can be located in potential parameter space using only third manifold basis functions while 15 require fourth manifold functions. This is contrary to previous suggestions (Briels, 1980) that many manifolds (i.e. $\ell_{i}, \ell_{j}$ go up to ten or more) are needed to accurately describe tetrahedral molecules such as methane and adamantane. It is true that an unwieldly large number of parameters are needed if the parameters are determined using the orthogonality of the basis functions to project out each parameter through integration over its domain. This is the standard method for evaluating intermolecular potential parameters from an arbitrary intermolecular potential. The drawback to this method is that the integrations are dominated by contributions near singularities in the potential. Therefore the potential parameter values evaluated in this manner are strongly influenced by high-energy interactions between molecules that are insignificant in determining the free energy of the crystal. The low-energy interactions that dominate the free-energy calculation are insignificant in evaluating the IP parameters evaluated in this way. The result is a slowly convergent potential with many additive contributions in the vicinity of singularities and offsetting contributions elsewhere. What is needed for useful GPDs is a method for evaluating IP parameters from an arbitrary intermolecular potential that stresses the important lowenergy interactions and results in large, but not necessarily infinite, energies in the vicinity of singularities (Missaghi et al., 2010). Current work is a proof by demonstration. 
Truncation of the intermolecular potential after the fourth manifold leaves up to 15 potential parameters, which is far fewer than the previous assertions but still too many to easily visualize. Software such as $G G O B I$ facilitates investigations of high-dimensional spaces in an interactive way (Lang, 2003). Any number of axes may be displayed and spatial relationships between the RPs analyzed using the software.

Interpreting the potential coefficients in Table 3 is analogous to describing multipole electrostatic interactions. These potential coefficients include contributions from all interaction modes and are not limited to electrostatic interactions, however. In the case of tetrahedral molecules the octopole $\left(\ell_{i}=3\right)$ is the first non-zero multipole. Coefficients such as $v_{0,3,3}$ and $v_{0,4,4}$ where $\ell_{i}$ or $\ell_{j}$ is zero represent an octopole or hexadecapole of one molecule interacting with the zeroth pole of its neighbors. They are the crystal-field coefficients. As the basis functions may also be used as quantum basis sets, $\boldsymbol{v}$ may be calculated $a b$ initio and has been given physical interpretations via symmetry-adapted perturbation theory (van der Avoird et al., 1994). For instance, although dispersion and induction forces can be found in all components of $\boldsymbol{v}$, electrostatic forces contribute only to $v_{\ell_{i}, \ell_{i}+\ell_{j}, \ell_{j}}^{n_{n}, n_{\mu}}$ (Stone \& Tough, 1984) such as $v_{3,6,3}, v_{3,7,4}$ and $v_{4,8,4}$.

One drawback of using the algorithm in $\$ 2.2$ is that, by finding the maximum energy difference between a phase and all others, the RPs of neighboring phases tend to be spread apart. This is because an RP is a vector representing a region in a space, not a unique set of IP parameters. Homologous series of molecules (i.e. $\mathrm{CF}_{4}, \mathrm{CCl}_{4}, \mathrm{CBr}_{4}, \mathrm{CI}_{4}$ ) are expected to show trends in $\boldsymbol{v}$ space. If members of homologous series have different crystal structures, then they have different RPs. However, different RPs are widely spread by our algorithm. Therefore RPs of homologous series are often widely spaced, even if the molecules are expected to have similar intermolecular potentials. This hides the expected trends among homologues. Other methods are required to further constrain the RPs assigned to individual molecules before trends among homologous series can be investigated using GPDs.

Correlations are evident in reference-lattice assignments. An example of this is a series of molecules with molecular formula $\mathrm{C}_{16} \mathrm{H}_{36} X_{4} \mathrm{In}_{4} \mathrm{~N}_{4}$ where $X$ is $\mathrm{Cl}, \mathrm{Br}$ or I. The first two structures, $14 e$ MECKUA and $12 i$ MECKOU, pack in an f.c.c. reference lattice while the last, 11e MECKIO, packs in a tetragonal reference lattice intermediate between f.c.c. and b.c.c. which is slightly closer to the b.c.c. reference lattice. This indicates at least the translational part of homologue potentials is similar and that small changes in the atomic constituency can slightly alter the IP, but significantly alter crystal structure. This is consistent with experience that similar molecules often have very different crystal structures despite similar intermolecular potentials. GPDs acknowledge this by placing the seemingly disparate structures close to one another in IP parameter space.

In McClurg \& Keith (2010) we noted that structures $195 a$ and $197 a$ were very similar to higher-symmetry structures (215a and 217a). If the molecules remained tetrahedral then the crystal would retain higher symmetry but the reported atomic coordinates indicate a minor molecular distortion reducing the space-group symmetry. Assuming that the reported space group is correct, these structures require a symmetry-breaking pathway from s.c. and b.c.c. with a minimal truncation manifold of $\ell_{i}^{\max }=6$ or 9 (depending on the potential-dependent transition pathway taken). In contrast to these high manifold requirements most pathways require a third or fourth manifold basis set. In view of the success of finding RPs using only the two first manifolds $\left(\ell_{i}^{\max }=3\right.$ or 4$)$ for all other molecules in the data set, it seems unlikely that such a large number of rapidly oscillating basis functions would be required to properly describe the intermolecular potential for these structures. It seems more likely that, barring a Jahn-Teller crystal distortion, the crystal symmetry may have been underspecified when reported to the CSD as suggested by McClurg \& Keith (2010) on different grounds.

\subsection{Extensions and features of the methodology}

We now discuss some of the ways to improve the model and algorithms. An issue affecting the numerical accuracy of the RP is how large a library of alternate crystal structures is needed to localize the RP in $\boldsymbol{v}$ space. In our previous work (Mettes et al., 2004) we considered just the high-symmetry point isotropy subgroups and in this work have followed suit since these are the most common (Stokes \& Hatch, 1988). Recall that space-group IRs are indexed by reciprocal-space vectors (Kovalev et al., 1993; Zak et al., 1969). There are the same number of $\mathbf{k}$ points in reciprocal space as there are unit cells in the crystal and there is a correspondence between $\mathbf{k}$ points and supercell patterns in the crystal. Experimental structures are supercells in their reference lattice. As nearly all experimental structures contain a relatively small number of clustered parent-lattice unit cells as sublattices, symmetry breaking occurs at $\mathbf{k}$ vectors corresponding to this small cluster. These are $\mathbf{k}$ vectors at high-symmetry points in the reference-lattice Brillouin zone. If the experimental structure has a unit cell which is large or flattened/elongated, however, $\mathbf{k}$ vectors corresponding to this larger or longer/flatter group of parent-lattice unit cells will be on high-symmetry lines and planes. Symmetry-breaking pathways for experimental lattices pertaining to b.c.c. and f.c.c. reference lattices in Appendix $A$ show that some experimental structures contain large [i.e. CTBROM (More et al., 1977)] or non-clustered parent-lattice unit cells [i.e. MTRETC10 (Harrison et al., 1972)] and their $\mathbf{k}$ vectors therefore are from high-symmetry plane and line IRs. Although these cases are less common, such isotropy subgroups could be included in the candidate lattices in $\$ 2.2 .3$. Providing such additional structures in the library would place additional constraints on the RP for the observed structures and therefore would further localize the RP for each observed structure at the expense of a much larger library.

In $\S 2.2 .3$ we chose to consider only isotropy subgroups with one occupied Wyckoff point. This is a commonly used simplification (Verwer \& Leusen, 1998). We have also discarded coupled IR isotropy subgroups for the same reason. Our method could be extended to include library structures 
with multiple Wyckoff points and those from coupled IRs, although the computational demands are much higher because of the larger set of candidate lattices and so we do not pursue it here. The effect would be to further localize the RPs of each phase, at the expense of a much larger library.

Although tetrahedral molecules are used in the current example, any molecular point group could be used without a dramatic increase in the number of potential coefficients $v$ in the first two non-trivial manifolds. This is because the absence of IP coefficients in lower manifolds for high-symmetry molecules is offset by the larger number of parameters in higher manifolds. Consider the number of basis functions in the first two non-trivial manifolds of $I_{h}$ (the icosahedral group) and $C_{1}$ (the point group of no special symmetry). These are the highest and lowest molecular point-group symmetries, respectively. The first three manifolds of $I_{h}$ containing a totally symmetric molecular representation are the zeroth, sixth and tenth manifolds. The number of potential coefficients is seven on the sixth manifold, $\left\{v_{6,0,6}^{1,1}, v_{6,2,6}^{1,1}, \ldots, v_{6,12,6}^{1,1}\right\}, 11$ on the tenth manifold, $\left\{v_{10,0,1}^{1,1}, v_{10,2,10}^{1,1}, \ldots, v_{10,20,10}^{1,1}\right\}$, and seven for the cross manifolds, $\left\{v_{6,4,10}^{1,1}, v_{6,6,10}^{1,1}, \ldots, v_{6,16,10}^{1,1}\right\}$. There are also two crystal-field coefficients, $\left\{v_{0,6,6}^{1,1}, v_{0,10,10}^{1,1}\right\}$, giving a total of 27 potential coefficients for $I_{h}$. For $C_{1}$ the first three manifolds are the zeroth, first and second. The coefficients on the first manifold are $\left\{v_{1,0,1}^{n_{\sigma}, n_{\mu}}, v_{1,2,1}^{n_{\sigma}, n_{\mu}}\right\}$. Although there are three copies of the totally symmetric molecular representation on the first manifold and therefore the molecular-frame indices $\sigma$ and $\mu$ in equation (1) go over $\{1,2,3\}$, we are free to choose the standard orientation for the molecule corresponding to Euler angles $\{0,0,0\}$. If it is chosen with the IP major axis parallel to the laboratory $z$ axis then only one of these is non-zero. This leaves two coefficients, $\left\{v_{1,0,1}^{1,1}, v_{1,2,1}^{1,1}\right\}$. If the molecular minor axis in the standard orientation is oriented parallel to the laboratory $x$ axis, only four of the $\sigma$ and $\mu$ are non-zero in the second manifold. This leaves 48 coefficients on the second manifold, $\left\{v_{2,0,2}^{n_{\sigma}, n_{\mu}}, v_{2,2,2}^{n_{\sigma}, n_{\mu}}, v_{2,4,2}^{n_{\sigma}, n_{\mu}}\right\}$. With five additional crystalfield coefficients $\left\{v_{0,1,1}^{1,1}, v_{0,2,2}^{1, n}\right\}$ and eight cross-manifold coefficients $\left\{v_{1,1,2}^{1, n_{\mu}}, v_{1,3,2}^{1, n_{\mu}}\right\}$ the total number of coefficients on the first three manifolds of $C_{1}$ is 60 , an increase of roughly twofold from highly symmetrical $I_{h}$. This shows that although this method has been applied to tetrahedral molecules it is applicable to other molecular point-group symmetries with only a modest change in the number of parameters.

Throughout we have implemented a simple direct cut-off truncation scheme in which a doubly infinite summation is cut off at a maximum manifold number $\ell_{i}^{\max }$. An alternative is the manifold sum cut-off in which we truncate such that $\ell_{i}+\ell_{j} \leq \ell^{\max }$. This truncation would include smoother functions before more rapidly oscillating ones, which is consistent with our expectations of lower-energy electronic contributions to the potential. Also the number of potential parameters increases at a slower rate with this truncation. This is shown in Table 4 for both truncation schemes, where we compare the cumulative number of parameters for the $C_{1}, T_{d}$ and $I_{h}$ point groups at different manifolds. The truncation method used in this study is a square truncation of the double sum while the alternative is a triangular truncation. The manifold sum
Table 4

Comparison of the cumulative number of parameters in the potential for a truncation at a given manifold for two truncation strategies and three molecular point groups.

\begin{tabular}{|c|c|c|c|c|c|c|c|}
\hline \multicolumn{4}{|c|}{ Direct truncation } & \multicolumn{4}{|c|}{$\ell$-sum truncation } \\
\hline$\ell_{i}$ & $C_{1}$ & $T_{d}$ & $I_{h}$ & $\ell_{i}+\ell_{j}$ & $C_{1}$ & $T_{d}$ & $I_{h}$ \\
\hline 0 & $\dagger$ & $\dagger$ & $\dagger$ & 0 & $\dagger$ & $\dagger$ & $\dagger$ \\
\hline 1 & 3 & 0 & 0 & 1 & 1 & 0 & 0 \\
\hline 2 & 60 & 0 & 0 & 2 & 6 & 0 & 0 \\
\hline 3 & & 5 & 0 & 3 & 15 & 1 & 0 \\
\hline 4 & & 10 & 0 & 4 & & 1 & 0 \\
\hline 6 & & & 8 & 6 & & 6 & 1 \\
\hline 10 & & & 19 & 10 & & & 8 \\
\hline
\end{tabular}

$\dagger$ There is one isotropic basis function for $\ell_{i}=\ell_{j}=0$ in each case, but it does not drive orientational ordering.

truncation adds new parameters into the potential more slowly than single manifold truncation. Therefore, the 15 coefficients used here are sufficient but may not be necessary. Further investigation of global phase diagrams with $\ell$-sum truncation is needed to test this hypothesis. This is important since lowerdimensional GPDs would be easier to construct and to use.

From the foregoing discussion we have seen that 15 coefficients are sufficient to locate our data set of experimental structures in potential parameter space. We have not investigated whether a linear combination would be better. Principal component analysis could be used to identify linear combinations of basis functions that better fit molecules. It is possible that fewer than 15 coefficients are necessary.

An analog in the physics community to molecular crystal GPDs are the various Ising-type GPDs, such as axial nextnearest-neighbor Ising (ANNNI) GPDs. Despite their simple potentials, these have been used for decades to explore a rich variety of commensurate and incommensurate phases (Gendiar \& Nishino, 2005; Kasama et al., 2006; Muraoka et al., 1999; Sato \& Matsubara, 1999; Kim et al., 1995) that can be applied to realistic materials such as silicon carbide, titanium aluminium, group-IV element polytypes, and many others (Wong-Leung et al., 2005; Colinet \& Pasturel, 2002; Raffy et al., 2002). These differ from molecular crystal GPDs in that their basis functions are binary variables $S_{i} \in\{1,-1\}$ coupled by a constant $J$, although such couplings typically exist with nextnearest neighbors or larger. It will be interesting to compare the results of these simplified models with a full molecular crystal GPD in our upcoming work (Keith et al., 2010).

A further extension would be to include the intersection of each solid GPD with a fluid GPD. This would not only allow crystallographers to identify the first phase to precipitate from solution, but would also allow inclusion of specific crystallization conditions and kinetic effects. Thus the calculation of the exact location of the liquid/solid surface could be performed with as complex or simple a crystal-growth model as one desires, using not only consideration of surface free energies, crystal habit and surface defect structure, but also external conditions such as undercooling, seeding etc. In theory even kinetic barriers to crystallization could be estimated between solution and GPD candidate phases, thus suggesting a more realistic progression of polymorph crystal- 
lization since GPDs find not only the thermodynamic ground state but also all metastable states.

\subsection{Conclusions}

We have shown that a molecular crystal GPD with a small number of reference lattices derived by McClurg \& Keith (2010) can summarize the experimental data using a modest number of IP parameters. The data set is diverse enough to test the GPD's ability to classify a wide range of space groups using a common intermolecular potential. Just as the van Konyenburg GPD classification based on the simple van der Waals equation of state is nonetheless widely used to classify the phase behavior of real binary mixtures, molecular crystal global phase diagrams may be useful in elucidating phase behavior of a variety of real substances and, in turn, used to develop novel intermolecular potentials and materials. Further work to apply these results in constructing temperature-dependent GPDs is needed and is underway.

\section{APPENDIX $A$}

\section{Detailed derivation of equation (1)}

We choose to expand the potential using a complete set of two-molecule basis functions that span the rotational space $S O(3)$ parameterized by Euler angles $\omega$ of molecule $i, j$ and their intervening orientational space $S^{2}$ parameterized by solid angle $\boldsymbol{\Omega}_{i j}=\left(\theta_{i j}, \varphi_{i j}\right)$. Coupling two $S O(3)$ irreducible representations (IRs) $D_{m_{i} n_{i}}^{\ell_{i}}\left(\omega_{i}\right)$ and $D_{m_{j} n_{j}}^{\ell_{j}}\left(\omega_{j}\right)$ and a spherical harmonic $C_{m}^{\ell}\left(\boldsymbol{\Omega}_{i j}\right)$ gives

$$
W_{\ell_{i} \ell_{j}}^{n_{i} n_{j}}=\sum_{m_{i} m m_{j}}\left(\begin{array}{ccc}
\ell_{i} & \ell & \ell_{j} \\
m_{i} & m & m_{j}
\end{array}\right) D_{m_{i} n_{i}}^{\ell_{i}}\left(\boldsymbol{\omega}_{i}\right) C_{m}^{\ell}\left(\boldsymbol{\Omega}_{i j}\right) D_{m_{j} n_{j}}^{\ell_{j}}\left(\boldsymbol{\omega}_{j}\right),
$$

where the explicit functional dependence of $C_{m}^{\ell}$ and $D_{m_{j} n_{j}}^{\ell_{j}}$ has been dropped. This is done in the following manner, where (Varshalovich et al., 1988)

$$
\begin{gathered}
D_{m n}^{\ell}\left(\boldsymbol{\omega}_{i}\right)=\exp \left(-i m \alpha_{i}\right) d_{m n}^{\ell}\left(\beta_{i}\right) \exp \left(-i n \gamma_{i}\right), \\
d_{m n}^{\ell}\left(\beta_{i}\right)=\sum_{k=\max (0, m-n)}^{\min (\ell+m, \ell-n)}(-1)^{k} \\
\times \frac{[(\ell+m) !(\ell-m) !(\ell+n) !(\ell-n) !]^{1 / 2}}{k !(\ell+m-k) !(\ell-n-k) !(n-m+k) !} \\
\times\left[\cos \left(\beta_{i} / 2\right)\right]^{2 \ell+m-n-2 k}\left[\sin \left(\beta_{i} / 2\right)\right]^{2 k-m+n}, \\
C_{m}^{\ell}\left(\boldsymbol{\Omega}_{i j}\right)=\left(\frac{4 \pi}{2 \ell+1}\right)^{1 / 2} Y_{m}^{\ell}\left(\mathbf{\Omega}_{i j}\right) ;
\end{gathered}
$$

we first couple angular basis functions of molecules $i$ and $j$,

$$
A_{m_{(i j)}}^{\ell_{(i j)}}=\sum_{m_{i} m_{j}} C_{\ell_{i} n_{i}, \ell_{j} m_{j}}^{\ell_{(i j} m_{(i)}} D_{m_{i} n_{i}}^{\ell_{i}} D_{m_{j} n_{j}}^{\ell_{j}},
$$

where $C_{\ell_{i} m_{i}, \ell_{i} m_{j}}^{\ell_{(i j)} m_{(j)}}$ is a Clebsch-Gordan coefficient (Varshalovich et al., 1988). Coupling $A_{m_{(i j)}}^{\ell_{(i j)}}$ to $C_{m}^{\ell}\left(\boldsymbol{\Omega}_{i j}\right)$ and requiring the overall state to be a scalar $W$, we obtain

$$
W_{\ell_{i} \ell \ell_{j}}^{n_{i} n_{j}}=\sum_{m m_{(i j)}} C_{\ell m, \ell_{(i j)}}^{00} m_{(i j)} A_{m_{(i j)}}^{\ell_{(i j)}} C_{m}^{\ell}
$$

By definition the Clebsch-Gordan coefficient $C_{\ell_{1} m_{1}, \ell_{2} m_{2}}^{\ell m}$ is zero unless $m_{1}+m_{2}=m$ so that $m_{2}=-m_{1}$ if $m=0$. It is also zero unless $\left|\ell_{1}-\ell_{2}\right| \leq \ell \leq \ell_{1}+\ell_{2}$, and, since $m_{1,2} \in\left\{\ell_{1,2} \ldots-\ell_{1,2}\right\}$, this implies that $\ell_{1}=\ell_{2}$. Thus $C_{\ell m, \ell_{(i j)} m_{(i i)}}^{00}$ in equation (15) simplifies to $C_{\ell m, \ell \bar{m}}^{00}$. With the identity (Varshalovich et al., 1988)

$$
C_{\ell m, \ell \bar{m}}^{00}=(-1)^{\ell+m} /(2 \ell+1)^{1 / 2}
$$

we have

$$
W_{\ell_{i} \ell_{j}}^{n_{i} n_{j}}=\sum_{m_{i} m m_{j}} C_{\ell_{i} m_{i}, \ell_{j} m_{j}}^{\ell \bar{m}} \frac{(-1)^{\ell+m}}{(2 \ell+1)^{1 / 2}} D_{m_{i} n_{i}}^{\ell_{i}} C_{m}^{\ell} D_{m_{j} n_{j}}^{\ell_{j}} .
$$

Likewise using the identity (Varshalovich et al., 1988)

$$
C_{\ell_{i} m_{i}, \ell_{j} m_{j}}^{\ell \bar{m}}=(-1)^{\ell_{i}-\ell_{j}-m}(2 \ell+1)^{1 / 2}\left(\begin{array}{ccc}
\ell_{i} & \ell_{j} & \ell \\
m_{i} & m_{j} & m
\end{array}\right)
$$

gives

$$
W_{\ell_{i} \ell \ell_{j}}^{n_{i} n_{j}}=\sum_{m_{i} m m_{j}}(-1)^{\ell_{i}-\ell_{j}+\ell}\left(\begin{array}{ccc}
\ell_{i} & \ell_{j} & \ell \\
m_{i} & m_{j} & m
\end{array}\right) D_{m_{i} n_{i}}^{\ell_{i}} C_{m}^{\ell} D_{m_{j} n_{j}}^{\ell_{j}} .
$$

To remove the phase factor one may exploit the mirror symmetry of the $3 j m$ symbol

$$
\left(\begin{array}{ccc}
\ell_{i} & \ell_{j} & \ell \\
m_{i} & m_{j} & m
\end{array}\right)=(-1)^{\ell_{i}+\ell_{j}+\ell}\left(\begin{array}{ccc}
\ell_{i} & \ell & \ell_{j} \\
m_{i} & m & m_{j}
\end{array}\right)
$$

leaving

$$
W_{\ell_{i} \ell \ell_{j}}^{n_{i} n_{j}}=\sum_{m_{i} m m_{j}}\left(\begin{array}{ccc}
\ell_{i} & \ell & \ell_{j} \\
m_{i} & m & m_{j}
\end{array}\right) D_{m_{i} n_{i}}^{\ell_{i}} C_{m}^{\ell} D_{m_{j} n_{j}}^{\ell_{j}}
$$

Equation (21) gives basis functions $W$ from equation (10) without any symmetry adaptation. The $W_{\ell_{i} \ell \ell_{j}}^{n_{i} n_{j}}$ form a complete set of orthogonal IP basis functions over the eight-dimensional space $S O(3) \times S^{2} \times S O(3)$. Similar but slightly different constructions are given in the literature (van der Avoird et al., 1980, 1994; Briels, 1980; Stone \& Tough, 1984).

While $W_{\ell_{i} \ell \ell_{j}}^{n_{i} n_{j}}$ are general, it is computationally advantageous to project out the point-group symmetry of the molecule and that of the Wyckoff point. Appendix $B$ reviews our use of projection operators which amounts to matrix multiplication by a sparse unitary matrix $S_{n_{i} n_{\sigma}}^{\ell_{i}}$ where $\sigma$ refers to a point-group IR and $n_{\sigma}$ is a particular component of the IR. For example, using projection operators for the molecular point group yields symmetry-adapted matrix elements

$$
T_{m_{i} n_{\sigma}}^{\ell_{i}}\left(\omega_{i}\right)=\sum_{n_{i}} D_{m_{i} n_{i}}^{\ell_{i}}\left(\omega_{i}\right) S_{n_{i} n_{\sigma}}^{\ell_{i}}
$$

which may be coupled to produce symmetry-adapted basis functions

$$
F_{\ell_{i} \ell \ell_{j}}^{n_{\sigma} n_{\mu}}=\sum_{m_{i} m m_{j}}\left(\begin{array}{ccc}
\ell_{i} & \ell & \ell_{j} \\
m_{i} & m & m_{j}
\end{array}\right) T_{m_{i} n_{\sigma}}^{\ell_{i}}\left(\boldsymbol{\omega}_{i}\right) C_{m}^{\ell}\left(\boldsymbol{\Omega}_{i j}\right) T_{m_{j} n_{\mu}}^{\ell_{j}}\left(\boldsymbol{\omega}_{j}\right) .
$$

With these basis functions the potential is 


$$
V=\frac{1}{2} \sum_{i j} \sum_{\ell_{i} \ell \ell_{j} n_{\sigma} n_{\mu}} v_{\ell_{i} \ell \ell_{j}}^{n_{\sigma} n_{\mu}}\left(r_{i j}\right) F_{\ell_{i} \ell \ell_{j}}^{n_{\sigma} n_{\mu}}\left(\boldsymbol{\omega}_{i}, \boldsymbol{\Omega}_{i j}, \boldsymbol{\omega}_{j}\right),
$$

where the one half avoids overcounting, $\ell_{i}, \ell_{j} \in \mathbb{N}$, $\left|\ell_{i}-\ell_{j}\right| \leq \ell \leq \ell_{i}+\ell_{j}$, and $\left.v_{\ell_{i}, \ell_{\ell}, \ell_{j}}^{n_{n}, r_{i j}}\right)$ are coefficients which are a function of the distance $r_{i j}$ between molecular centers. The full set of these in the potential is termed $\boldsymbol{v}$. Subscripts $\sigma, \mu$ are a compound index referring to multiple copies of the molecular point group unit IR subduced in the $\left(\ell_{i}, \ell_{j}\right)$ th manifold of $S O(3)$ and $n_{\sigma}, n_{\mu}$ is its dimension, which is always $n_{\sigma}, n_{\mu}=1$. Point-group IR subduction frequencies in spherical harmonics are discussed elsewhere (Bradley \& Cracknell, 1972). All other point-group IRs do not have the full molecular symmetry and so are zero to first order.

Projecting out Wyckoff point symmetry from the functions $T_{m_{i} n_{\sigma}}^{\ell_{i}}$ gives rotator functions

$$
U_{m_{\tau} n_{\sigma}}^{\ell_{i}}\left(\omega_{i}\right)=\sum_{m_{i} n_{i}} S_{m_{i} m_{\tau} *}^{\ell_{i}} D_{m_{i} n_{i}}^{\ell_{i}}\left(\omega_{i}\right) S_{n_{i} n_{\sigma}}^{\ell_{i}} .
$$

Expressing the potential using coupled rotator functions gives

$$
V=\frac{1}{2} \sum_{i j} \sum_{\ell_{i} \ell_{j} m_{\tau} m_{\rho} n_{\sigma} n_{\mu}} U_{m_{\tau} n_{\sigma}}^{\ell_{i}}\left(\boldsymbol{\omega}_{i}\right) J_{m_{\tau} n_{\sigma} m_{\rho} n_{\mu}}^{\ell_{i} \ell_{j}}\left(\boldsymbol{\Omega}_{i j}\right) U_{m_{\rho} n_{\mu}}^{\ell_{j}}\left(\boldsymbol{\omega}_{j}\right)
$$

where

$$
\begin{aligned}
J_{m_{\tau} n_{\sigma} m_{\rho} \ell_{\ell_{j}}}\left(\boldsymbol{\Omega}_{i j}\right)= & \sum_{\ell m_{i} m m_{j}} v_{\ell_{i}, \ell_{,} \ell_{j}}^{n_{\sigma}, n_{\mu}}\left(r_{i j}\right)\left(\begin{array}{ccc}
\ell_{i} & \ell & \ell_{j} \\
m_{i} & m & m_{j}
\end{array}\right) \\
& \times S_{m_{\tau} \ell_{i}}^{\ell_{i}} C_{m}^{\ell} S_{m_{\rho}}^{\ell_{j}} m_{j}
\end{aligned}
$$

is a dimensionless coupling function. Subscripts $\tau, \rho$ are a compound index referring to multiple copies of the Wyckoff point-group IRs subduced in the $\left(\ell_{i}, \ell_{j}\right)$ th manifold of $S O(3)$ and $m_{\tau}, m_{\rho}$ goes over the dimensions of each IR.

\section{APPENDIX $B$}

\section{Projection operators}

Symmetries of the molecule and Wyckoff point of the crystal exist within $D_{m_{i}, n_{i}}^{\ell_{i}}$ simultaneously and can be obtained by applying projection operators (Bradley \& Cracknell, 1972)

$$
P_{n_{\tau} n_{\tau}}^{\tau}=\left(d_{\tau} /|G|\right)^{1 / 2} \sum_{g \in G} D_{n_{\tau} n_{\tau}}^{\tau *}(g) g
$$

where $d_{\tau}$ is the dimension of the IR $\tau$ belonging to the group $G$ of order $|G|, \mathbf{D}^{\tau}$ is the matrix onto which the IR maps $g$, and subsequent orthonormalization is occasionally required. We have used a slightly different normalization which decreases the computation in this orthonormalization. Acting upon the elements $D_{m n}^{\ell}$ gives

$$
P_{n_{\tau} n_{\tau}}^{\tau} \circ D_{m n}^{\ell}=\sum_{n} D_{m n}^{\ell} S_{n n_{\tau}}^{\ell}
$$

producing a linear combination with the symmetry of $\tau$. The coefficients $S_{n n_{\tau}}^{\ell}$ form a sparse unitary matrix.
This work received financial support from the American Chemical Society Petroleum Research Fund (PRF \#41774AC10), DOE grant No. DE-FG02-03ER46059 and NSF grant DMR-0520547. Computational resources maintained by the University of Minnesota Supercomputer Institute were used for portions of this research.

\section{References}

Allen, F. H. \& Motherwell, W. D. S. (2002). Acta Cryst. B58, 407-422. Aparicio-Martinez, S. \& Hall, K. R. (2007a). Ind. Eng. Chem. Res. 46, 273-284.

Aparicio-Martinez, S. \& Hall, K. R. (2007b). Ind. Eng. Chem. Res. 46, 285-290.

Avoird, A. van der, Wormer, P. E. S. \& Moszynski, R. (1994). Chem. Rev. 94, 1931-1974.

Avoird, A. van der, Wormer, P. E. S., Mulder, F. \& Berns, R. M. (1980). Topics Curr. Chem. 93, 1-50.

Bolz, A., Deiters, U. K., Peters, C. J. \& de Loos, T. W. (1998). Pure Appl. Chem. 70, 2233.

Bradley, C. J. \& Cracknell, A. P. (1972). The Mathematical Theory of Symmetry in Solids. Oxford University Press.

Braga, D., Grepioni, F. \& Orpen, A. G. (1999). Editors. Crystal Engineering: From Molecules and Crystals to Materials. Boston: Kluwer Academic.

Briels, W. J. (1980). J. Chem. Phys. 73, 1850-1861.

Cismondi, M. \& Michelsen, M. L. (2007). J. Supercrit. Fluid. 39, $287-$ 295.

Colinet, C. \& Pasturel, A. (2002). Intermetallics, 10, 751.

Desiraju, G. R. (1989). Crystal Engineering: The Design of Organic Solids. New York: Elsevier.

Gavezzotti, A. (2007). Molecular Aggregation: Structure Analysis and Molecular Simulation of Crystals and Liquids. Oxford University Press.

Gdanitz, R. J. (1997). Theoretical Aspects and Computer Modeling of the Molecular Solid State, edited by A. Gavezzotti, pp. 185-201. New York: Wiley.

Gendiar, A. \& Nishino, T. (2005). Phys. Rev. B, 71, 024404.

Gražulis, S., Chateigner, D., Downs, R. T., Yokochi, A. T., Quirós, M., Lutterotti, L., Manakova, E., Butkus, J., Moeck, P. \& Le Bail, A. (2009). J. Appl. Cryst. 42, 726-729.

Harrison, W., Marsh, W. \& Trotter, J. (1972). J. Chem. Soc. Dalton Trans. pp. 1009.

Hloucha, M., Lodge, J., Lenhoff, A. \& Sandler, S. (2001). J. Cryst. Growth, 64, 060104(R).

Holland, J. H. (1992). Adaptation in Natural and Artificial Systems: An Introductory Analysis with Applications to Biology, Control, and Artificial Intelligence. Cambridge: MIT Press.

Holman, K. T., Pivovar, A. M., Swift, J. A. \& Ward, M. D. (2001). Acc. Chem. Res. 34, 107-118.

James, H. M. \& Keenan, T. A. (1959). J. Chem. Phys. 31, 12-41.

Kasama, T., Muraoka, Y. \& Idogaki, T. (2006). Phys. Rev. B, 73, 214411.

Keith, J. B., McClurg, R. B. \& Fultz, B. (2010). In preparation.

Keith, J. B., Mettes, J. A. \& McClurg, R. B. (2004). Cryst. Growth Des. 4, 1009-1012.

Kim, J.-J., Mori, S. \& Harada, I. (1995). Phys. Lett. A, 202, 68-72.

Kirkpatrick, S., Gelatt, C. Jr \& Vecchi, M. (1983). Science, 220, 671680.

Konyenburg, P. H. van \& Scott, R. L. (1980). Philos. Trans. R. Soc. London Ser. A, 298, 495-540.

Kovalev, O. V., Worthey, G. C., Stokes, H. T. \& Hatch, D. M. (1993). Representation of Crystallographic Space Groups: Irreducible Representations, Induced Representation and Corepresentations. Berkshire: Gordon and Breach.

Lamoen, D. \& Michel, K. H. (1999). Phase Transit. 67, 789-807. 
Lang, D. T. (2003). Ggobi, http://www.ggobi.org/.

Lin, J. Y. Y. \& Keith, J. B. (2009). Virtual neutron facility, http:// vnf.caltech.edu/.

Lommerse, J. P. M., Motherwell, W. D. S., Ammon, H. L., Dunitz, J. D., Gavezzotti, A., Hofmann, D. W. M., Leusen, F. J. J., Mooij, W. T. M., Price, S. L., Schweizer, B., Schmidt, M. U., van Eijck, B. P., Verwer, P. \& Williams, D. E. (2000). Acta Cryst. B56, 697-714.

Lynden-Bell, R. M. \& Michel, K. H. (1994). Rev. Mod. Phys. 66, 721762.

McClurg, R. B. \& Keith, J. B. (2010). Acta Cryst. A66, 38-49.

Mettes, J. A., Keith, J. B. \& McClurg, R. B. (2004). Acta Cryst. A60, 621-636.

Michel, K. H., Copley, J. R. D. \& Neumann, D. A. (1992). Phys. Rev. Lett. 68, 2929-2932.

Missaghi, M. N., Mettes, J. A. \& McClurg, R. B. (2010). In preparation.

More, M., Baert, F. \& Lefebvre, J. (1977). Acta Cryst. B33, 3681-3684.

Moulton, B. \& Zaworotko, M. J. (2001). Chem. Rev. 101, 1629-1658.

Muraoka, Y., Oda, K. \& Idogaki, T. (1999). J. Magn. Magn. Mater. 195, 156-167.

Neumann, M. A., Press, W., Noldeke, C., Asmussen, B., Prager, M. \& Ibberson, R. M. (2003). J. Chem. Phys. 119, 1586-1589.

Padmaja, N., Ramakumar, S. \& Viswamitra, M. A. (1990). Acta Cryst. A46, 725-730.

Pelt, A. van, Peters, C. J., Swaan-Arons, J. \& Dieters, U. K. (1995). J. Chem. Phys. 102, 3361.

Polishuk, I., Wisniak, J. \& Segura, H. (2002). Phys. Chem. Chem. Phys. 4, 879-883.
Polishuk, I., Wisniak, J., Segura, H., Yelash, L. V. \& Kraska, T. (2000). Fluid Phase Equilib. 172, 1-26.

Raffy, C., Furthmuller, J. \& Bechstedt, F. (2002). Phys. Rev. B, 66, 075201.

Sato, A. \& Matsubara, F. (1999). Phys. Rev. B, 60, 10316.

Schmalzried, H. (2003). Z. Phys. Chem. 217, 1281-1302.

Sherwood, J. N. (1979). The Plastically Crystalline State. New York: Wiley.

Simon, J. \& Bassoul, P. (2000). Design of Molecular Materials: Supramolecular Engineering. New York: John Wiley and Sons.

Stokes, H. T. \& Hatch, D. M. (1988). Isotropy Subgroups of the 230 Crystallographic Space Groups. Teaneck: World Scientific.

Stokes, H. T. \& Hatch, D. M. (2002). Phys. Rev. B, 65, 144114.

Stokes, H. T., Hatch, D. M. \& Campbell, B. J. (2007). Isotropy, http:// stokes.byu.edu/isotropy.html.

Stokes, H. T., Hatch, D. M. \& Wells, J. D. (1991). Phys. Rev. B, 43, 11010-11018.

Stone, A. J. \& Tough, R. J. A. (1984). Chem. Phys. Lett. 110, 123-129.

Thalladi, V. R., Goud, B. S., Hoy, V. J., Allen, F. H., Howard, J. A. K. \& Desiraju, G. R. (1996). Chem. Commun. pp. 401-402.

Varshalovich, D. A., Moskalev, A. N. \& Khersonskii, V. K. (1988). Quantum Theory of Angular Momentum. Teaneck: World Scientific.

Verwer, P. \& Leusen, F. J. J. (1998). Rev. Comput. Chem. 12, 327-366.

Wong-Leung, J., Linnarsson, M. K., Svensson, B. G. \& Cockayne, D. J. H. (2005). Phys. Rev. B, 71, 165210.

Zak, J., Casher, H., Gluck, H. \& Gur, Y. (1969). The Irreducible Representations of Space Groups. New York: Benjamin. 